\title{
TOPOLOGICAL METHODS IN 3-DIMENSIONAL CONTACT GEOMETRY
}

An illustrated introduction to Giroux's convex surfaces theory

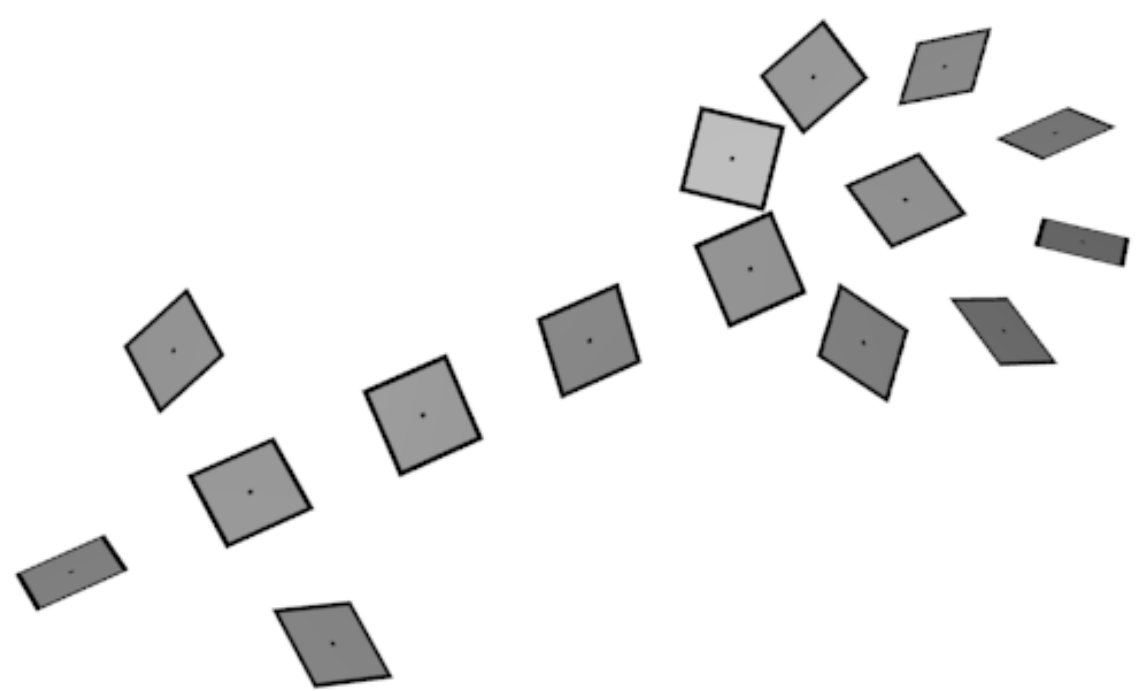

Patrick MASsOT

Notes for the Nantes summer school in

Contact and Symplectic Topology

June 2011

Revised in February 2013 


\section{Introduction}

These lecture notes are an introduction to the study of global properties of contact structures on 3-manifolds using topological rather than analytical methods. From that perspective, the main tool to study a contact manifold $(V, \xi)$ is the study of its $\xi$-convex surfaces. These surfaces embedded in $V$ are useful because all the information about $\xi$ near each of them is encoded into a surprisingly small combinatorial data. In order to illustrate the power of $\xi$-convex surfaces without long developments, we use them to reprove, following Giroux Gir00, two important theorems which were originally proved using different techniques by Bennequin Ben83 and Eliashberg Eli92.

Besides Giroux's original papers Gir91, Gir00, there are already two sets of lectures notes by Etnyre [Etn04] and Honda [Hon] and a book by Geiges Gei08] which cover almost all topics we will discuss as well as more advanced topics. Our goal is not to replace those references but to complement them. Mostly, we include many pictures that are not easily found in print and can help to build intuition. We focus on a small set of contact manifolds and illustrate all phenomena on those examples by showing explicit embedded surfaces. On the other hand, we almost never give complete proofs.

Chapter 1 explains the local theory of contact structures starting with the most basic definitions. There are many ways to define contact structures and contact forms and we use unusual geometric definitions in order to complement existing sources. We also try to explain the geometric intuition behind the theorems of Darboux-Pfaff and Gray rather than using Moser's path method without explanation.

Once enough definitions are given, an interlude states the theorems of Bennequin and Eliashberg that are proved at the high point of these notes. It serves as motivation for the rather long developments of Chapter 2.

Chapter 2 begins the study of surfaces in contact manifolds. The starting point is the singular foliation printed by a contact structure on any surface. We then work towards $\xi$-convex surfaces theory by simplifying gradually the contact condition near a surface. Once the amazing realization lemma is proved, we investigate obstructions to $\xi$-convexity and prove these obstructions are generically not present. The last section of this chapter then get the first fruits of this study by proving the Eliashberg-Bennequin inequalities.

Chapter 3 goes beyond the study of a single surface by studying some oneparameter families of surfaces. In particular we describe what happens exactly when one of the obstructions to $\xi$-convexity discussed in the preceding chapter arises. This allows us to prove the theorems of Bennequin and Eliashberg mentioned above. Until now, the proof of Bennequin's theorem using $\xi$-convex surfaces was explained only in Gir00. 
Of course this is only the beginning of a story which continues both by itself and in combination with holomorphic curves techniques.

Conventions: A plane field $\xi$ on a 3 -manifold $V$ is a (smooth) map associating to each point $p$ of $V$ a 2-dimensional subspace $\xi(p)$ of $T_{p} V$. All plane fields considered here will be coorientable, it means one can continuously choose one of the half spaces cut out by $\xi(p)$ in $T_{p} V$. In this situation, $\xi$ can be defined as the kernel of some nowhere vanishing 1 -form $\alpha: \xi(p)=\operatorname{ker} \alpha(p)$. The coorientation is given by the sign of $\alpha$. We will always assume that $V$ is oriented. In this situation a coorientation of $\xi$ combines with the ambient orientation to give an orientation on $\xi$. All contact structures in these notes will be cooriented.

Occasionally, we will include remarks or comments that are not part of the main flow of explanations. These remarks are typeset in small italic print. 


\section{Chapter 1}

\section{Local theory}

\subsection{Contact structures as rotating plane fields}

\subsubsection{The canonical contact structure on the space of con- tact elements}

Let $S$ be a surface and $\pi: S T^{*} S \rightarrow S$ the bundle of cooriented lines tangent to $S$ (also called contact elements for $S$ ). It can be seen as the bundle of rays in $T^{*} S$, hence the notation. The canonical contact structure on $S T^{*} S$ at a point $d$ is defined as the inverse image under $\pi_{*}$ of $d \subset T_{\pi(d)} S$, see Figure 1.1.

Suppose first that $S$ is the torus $T^{2}=\mathbb{R}^{2} / 2 \pi \mathbb{Z}^{2}$. Let $x$ and $y$ be the canonical $\mathbb{S}^{1}$-valued coordinates on $T^{2}$. A cooriented line tangent to $T^{2}$ at some point $(x, y)$ can be seen as the kernel of a $1-$ form $\lambda$ which has unit norm with respect to the canonical flat metric. So there is some angle $z$ such that $\lambda=\cos (z) d x-$ $\sin (z) d y$. Hence we have a natural identification of $S T^{*} T^{2}$ with $T^{3}$. In addition the canonical contact structure can be defined by $\cos (z) d x-\sin (z) d y$ now seen as a 1 -form on $T^{3}$ called the canonical contact form on $T^{3}$, see Figure 1.2 .

When $S$ is the sphere $\mathbb{S}^{2}, S T^{*} S$ is endowed with a free transitive action of $\mathrm{SO}_{3}(\mathbb{R})$ so it is diffeomorphic to $\mathrm{SO}_{3}(\mathbb{R})$. So there is a two-fold covering map
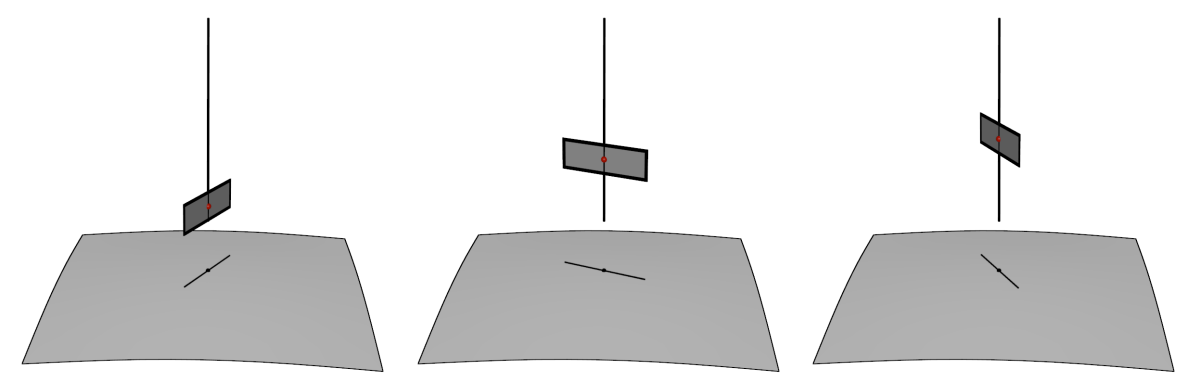

Figure 1.1: Canonical contact structure on the bundle of cooriented lines. At bottom is a portion of $S$ with a tangent line at some point. Above that point one gets the fiber by gluing top and bottom of the interval. The contact structure is shown at the point of the fiber corresponding to the line drawn below. 


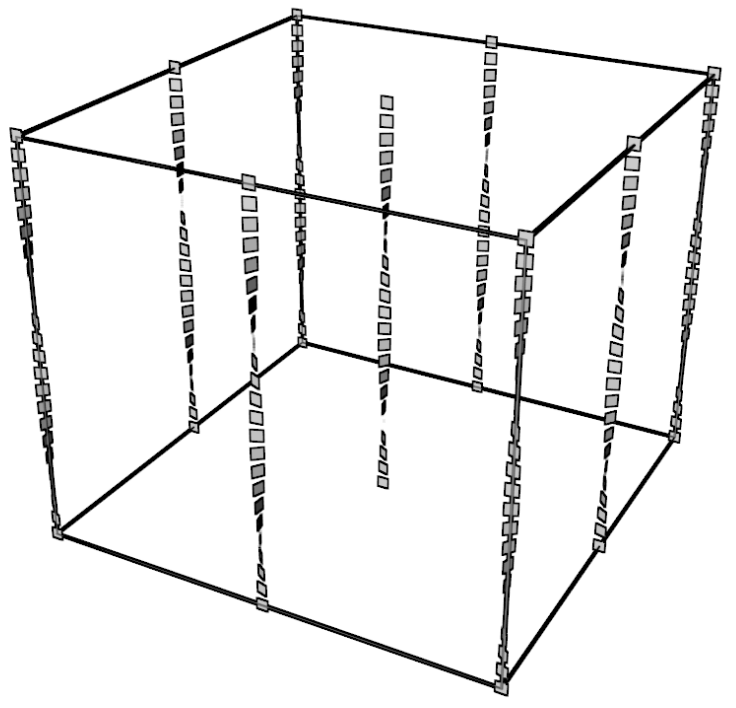

Figure 1.2: Canonical contact structure on $T^{3}$. Opposite faces of the cube are glued to get $T^{3}$

from $\mathbb{S}^{3} \simeq \mathrm{SU}(2)$ to $S T^{*} \mathbb{S}^{2}$. The lifted plane field is called the canonical contact structure on $\mathbb{S}^{3}$. We will see different ways of describing this example later on.

\subsubsection{Contact structures and contact forms}

Definition 1. A contact structure on a 3-manifold is a plane field which is locally diffeomorphic to the canonical contact structure on $S T^{*} T^{2}$. A contact form is a 1-form whose kernel is a contact structure. A curve or a vector field is Legendrian if it is tangent to a given contact structure.

As noted above all our manifolds will be oriented and diffeomorphisms in the above definition shall preserve orientations.

Theorem 2 (Darboux-Pfaff theorem). A 1-form $\alpha$ is a contact form if and only if $\alpha \wedge d \alpha$ is a positive volume form.

Let $\xi$ be the kernel of $\alpha$. The condition $\alpha \wedge d \alpha>0$ will henceforth be called the contact condition for $\alpha$. It is equivalent to the requirement that $d \alpha_{\mid \xi}$ is non-degenerate and defines the orientation of $\xi$ coming from the orientation of the ambient manifold and the coorientation of $\xi$.

Proof. If $\xi$ is a contact structure then the image of $\alpha$ in the local model is $f \alpha_{0}$ where $f$ is some nowhere vanishing function and $\alpha_{0}=\cos (z) d x-\sin (z) d y$. So

$$
\begin{aligned}
\alpha \wedge d \alpha & =f \alpha_{0} \wedge\left(f d \alpha_{0}+d f \wedge \alpha_{0}\right)=f^{2} \alpha_{0} \wedge d \alpha_{0} \\
& =f^{2} d x \wedge d y \wedge d z
\end{aligned}
$$


which is a positive volume form. More generally the above computation proves that the contact condition for a nowhere vanishing one-form depends only on its kernel.

Conversely, suppose $\alpha \wedge d \alpha$ is positive. Let $p$ be a point in $M$. We want to construct a coordinate chart around $p$ such that $\xi=\operatorname{ker}(\cos (z) d x-\sin (z) d y)$. We first choose a small surface $S$ containing $p$ and transverse to $\xi$. Then we pick a non-singular vector field $X$ tangent to $S$ and $\xi$ near $p$ and a small curve $c$ in $S$ containing $p$ and transverse to $X$, see Figure 1.3 . Let $y$ be a coordinate
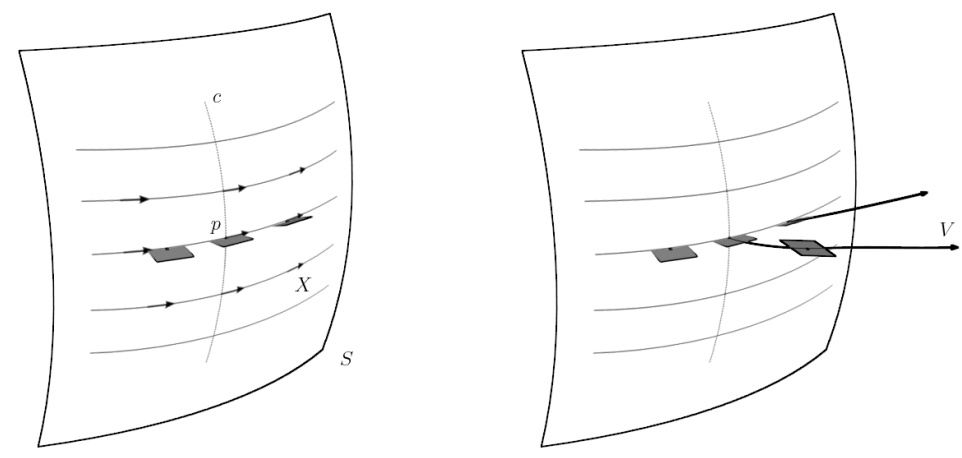

Figure 1.3: Proof of the Darboux-Pfaff theorem

on $c$. The flow of $X$ at time $x$ starting from $c$ gives coordinates $(x, y)$ on $S$ near $p$ in which $X=\partial_{x}$.

We now consider a vector field $V$ transverse to $S$ and tangent to $\xi$. The flow of $V$ at time $t$ starting from $S$ gives coordinates $(x, y, t)$ near $p$ such that $\alpha=f(x, y, t) d x+g(x, y, t) d y$ because $\alpha\left(\partial_{t}\right)=\alpha(V)=0$. Up to rescaling, one can use instead $\alpha_{1}=\cos z(x, y, t) d x-\sin z(x, y, t) d y$ for some function $z$ such that $z(x, y, 0)=0$. Now it is time to use the contact condition. We can compute

$$
\alpha_{1} \wedge d \alpha_{1}=\frac{\partial z}{\partial t} d x \wedge d y \wedge d t
$$

Remember the contact condition for $\alpha$ is equivalent to the contact condition for $\alpha_{1}$. So $\frac{\partial z}{\partial t}$ is positive and the implicit function theorem then guaranties that we can use $z$ as a coordinate instead of $t$.

In the above proof, $z(x, y, t)$ was the angle between $\xi$ and the horizontal $\partial_{x}$ is the plane normal to the Legendrian vector field $\partial_{t}$. We saw that the contact condition forces this angle to increase. This means that the contact structure rotates around $\partial_{t}$. The above proof essentially says that this rotation along Legendrian vector fields characterizes contact structures.

We now focus on the difference between contact structures and contact forms. The data of a contact form is equivalent to a contact structure and either a choice of a Reeb vector field or a section of its symplectization.

Definition 3. A Reeb vector field for a contact structure $\xi$ is a vector field which is transverse to $\xi$ and whose flow preserves $\xi$. 
If one has a Riemannian metric on a surface $S$ then the bundle of contact elements of $S$ can be identified with the unit tangent bundle STS and the geodesic flow is then the flow of a Reeb vector field for the canonical contact structure.

One can easily prove that each contact form $\alpha$ comes with a canonical Reeb vector field $R_{\alpha}$ which is characterized by $d \alpha\left(R_{\alpha}, \cdot\right)=0$ and $\alpha\left(R_{\alpha}\right)=1$. All Reeb vector fields arise this way.

Next, for any co-oriented plane field $\xi$ on a 3 -manifold $V$, one can consider the annihilator of $\xi$ in $T^{*} V$ :

$$
S_{\xi}:=\left\{\lambda \in T^{*} V \mid \operatorname{ker} \lambda=\xi \text { and } \lambda(v)>0 \text { if } v \text { is positively transverse to } \xi\right\} .
$$

It is a good exercise to check that a plane field $\xi$ on $V$ is a contact structure if and only if $S_{\xi}$ is a symplectic submanifold of $\left(T^{*} V, \omega_{\text {can }}\right)$. In this case $S_{\xi}$ is called the symplectization of $\xi$. The manifold $S_{\xi}$ is a principal $\mathbb{R}$-bundle where a real number $t$ acts by $\lambda \mapsto e^{t} \lambda$. Any contact form $\alpha$ is a section of this $\mathbb{R}$-bundle, and thus determines a trivialization $\mathbb{R} \times V \rightarrow S_{\xi}$ given by $(t, v) \mapsto e^{t} \alpha_{v}$. In this trivialization, the restriction of the canonical symplectic form $\omega_{\text {can }}$ becomes $d\left(e^{t} \alpha\right)$.

\subsection{Examples}

\section{The canonical contact structure on $\mathbb{R}^{3}$}

The universal cover of $S T^{*} T^{2}$ is of course $\mathbb{R}^{3}$ and the lifted contact structure is $\xi_{0}=\operatorname{ker}(\cos (z) d x-\sin (z) d y)$ where $x, y$ and $z$ are now honest real-valued coordinates. The plane field $\xi_{0}$ is called the standard contact structure on $\mathbb{R}^{3}$.

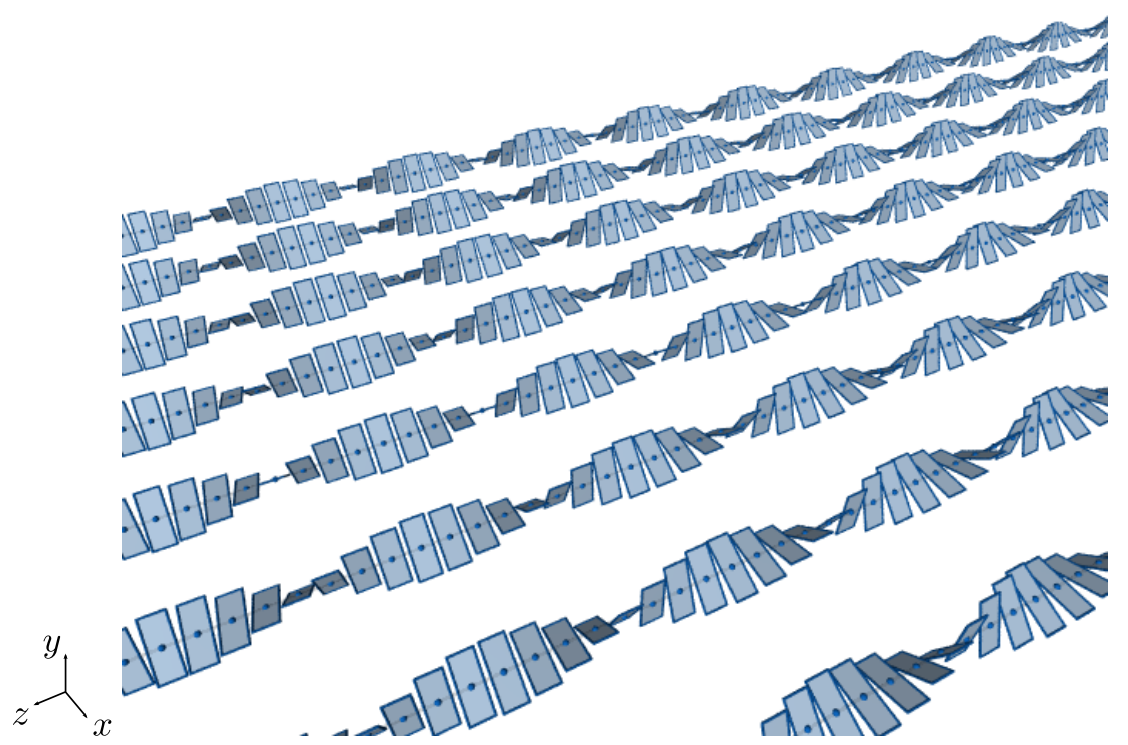

Figure 1.4: Universal cover of the standard contact structure on $\mathbb{T}^{3}$ seen from the side. It is invariant under translation in the vertical direction 
Depending on context, it can be useful to have different ways of looking at $\xi_{0}$ using various diffeomorphisms of $\mathbb{R}^{3}$. The image of $\xi_{0}$ under the diffeomorphism

$$
\left(\begin{array}{l}
x \\
y \\
z
\end{array}\right) \mapsto\left(\begin{array}{l}
t \\
p \\
q
\end{array}\right)=\left(\begin{array}{ccc}
\cos (z) & -\sin (z) & 0 \\
\sin (z) & \cos (z) & 0 \\
0 & 0 & 1
\end{array}\right)\left(\begin{array}{l}
x \\
y \\
z
\end{array}\right)
$$

is drawn in Figure 1.5. It admits the contact form $d t+p d q$ and arises naturally on $\mathbb{R}^{3}$ seen as the space of 1 -jets of functions from $\mathbb{R}$ to $\mathbb{R}$ (see e.g. Gei08, Example 2.5.11] for more information on this interpretation).

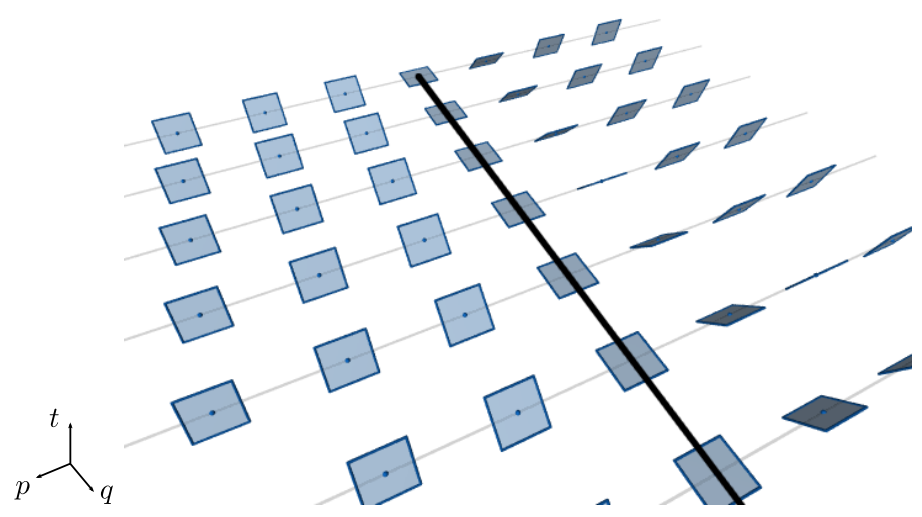

Figure 1.5: $\operatorname{ker}(d t+p d q)$ on $\mathbb{R}^{3}$. It is invariant under translation in the vertical direction. It becomes vertical only if one goes all the way to $p= \pm \infty$.

Figures 1.4 and 1.5 together are often confusing for beginners. First the thick black line $\{t=p=0\}$ in Figure 1.5 is Legendrian yet the contact structure does not seem to rotate along it. Second, it seems the two pictures exhibit Legendrian foliations by lines with very different behavior. In the second picture the contact structure turns half a turn along each leave whereas it turns infinitely many turns in the first picture.

Both puzzles are solved by the same picture. The diffeomorphism we used above sends the foliation by Legendrian lines of Figure 1.4 to a foliation containing the mysterious line $\{t=p=0\}$ in Figure 1.5 together with helices around that line, see Figure 1.6 .

So we first see where is the foliation of Figure 1.4 inside Figure 1.5. And second we remember that it makes sense to say that a plane field rotates along a curve only compared to something else. Contact structures rotate along Legendrian curves compared to neighborhood leaves of some Legendrian foliation. And indeed we see the contact structure turns infinitely many times along the mysterious line compared to the nearby Legendrian helices.

It is also sometimes convenient to consider the image of $\operatorname{ker}(d t+p d q)$ under the diffeomorphism $(t, p, q) \mapsto\left(q,-p, t+\frac{p q}{2}\right)$. This image is the kernel of $d z+$ $\frac{1}{2} r^{2} d \theta$ in cylindrical coordinates, see Figure 1.7. In this model, one sees clearly that, at each point, there are Legendrian curves going in every possible direction. 


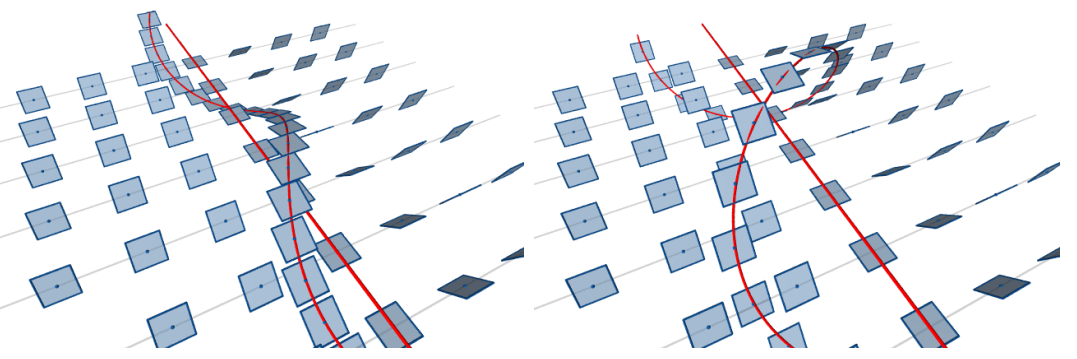

Figure 1.6: The mysterious line in Figure 1.5 together with two helices coming from the lines of Figure 1.4

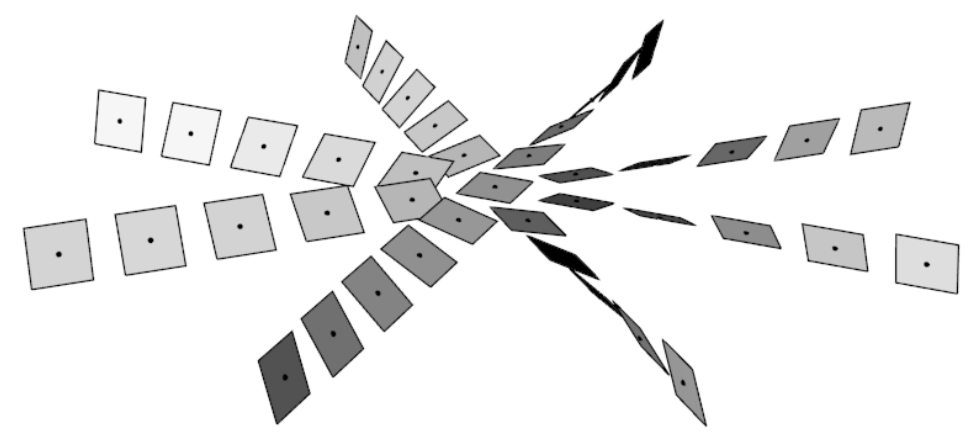

Figure 1.7: Another view of the standard contact structure on $\mathbb{R}^{3}$

Figure 1.8 shows how to deform Figure 1.7 to embed it inside Figure 1.5 .

Either of these contact structures (which are diffeomorphic by construction) will be called the canonical contact structure on $\mathbb{R}^{3}$. Of course they can all be used as the local model in the definition of a contact structure.

\section{The canonical contact structure on $\mathbb{S}^{3}$}

We have already met the canonical contact structure on $\mathbb{S}^{3}$ coming from the canonical contact structures on $S T^{*} \mathbb{S}^{2}$. One can prove that it is also

- the orthogonal of the Hopf circles for the round metric,

- a left-invariant contact structure on the Lie group $S U(2)$,

- $T \mathbb{S}^{3} \cap J T \mathbb{S}^{3}$ when $\mathbb{S}^{3}$ is seen as the boundary of the unit ball in $\mathbb{C}^{2}$ and $J$ denotes the action of multiplication by $i$ in $T \mathbb{C}^{2}$.

The complement of a point in the standard $\mathbb{S}^{3}$ is isomorphic to the standard $\mathbb{R}^{3}$, see Gei08, Proposition 2.1.8] for a computational proof valid in any dimension. 


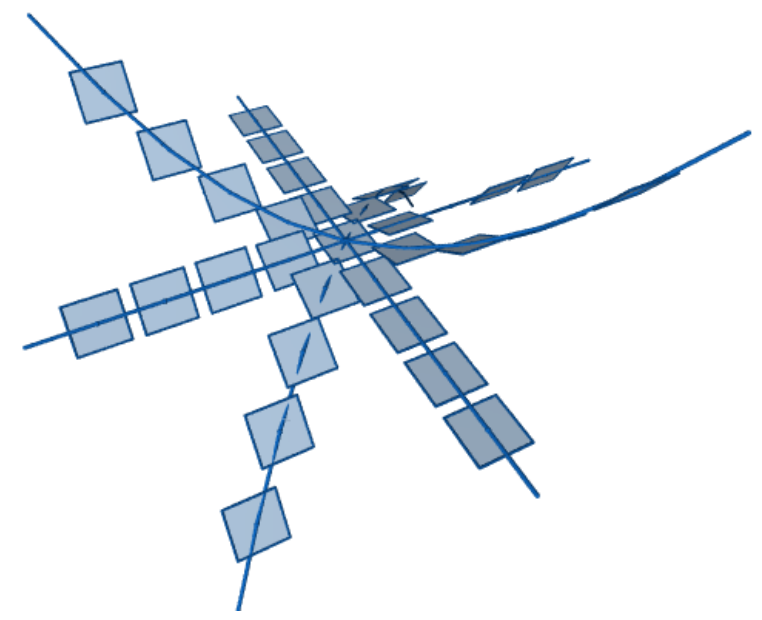

Figure 1.8: Embedding of Figure 1.7 into Figure 1.5

\section{$1.3 \quad$ Isotopies}

\subsubsection{Isotopic contact structures and Gray's theorem}

Up to now we considered two contact structures to be the same if they are conjugated by some diffeomorphism. One can restrict this by considering only diffeomorphisms corresponding to deformations of the ambient manifold. An isotopy is a family of diffeomorphisms $\varphi_{t}$ parametrized by $t \in[0,1]$ such that $(x, t) \mapsto \varphi_{t}(x)$ is smooth and $\varphi_{0}=I d$. The time-dependent vector field generating $\varphi_{t}$ is defined as $X_{t}=\frac{d}{d t} \varphi_{t}$. One says that two contact structures $\xi_{0}$ and $\xi_{1}$ are isotopic if there is an isotopy $\varphi_{t}$ such that $\xi_{1}=\left(\varphi_{1}\right)_{*} \xi_{0}$. In particular such contact structures can be connected by the path of contact structures $\xi_{t}:=\left(\varphi_{t}\right)_{*} \xi_{0}$. It is then natural to consider the seemingly weaker equivalence relation of homotopy among contact structures. The next theorem says in particular that, on closed manifolds, this equivalence relation is actually the same as the isotopy relation.

Theorem 4 (Gray [Gra59]). For any path $\left(\xi_{t}\right)_{t \in[0,1]}$ of contact structures on a closed manifold, there is an isotopy $\varphi_{t}$ such that $\varphi_{t}^{*} \xi_{t}=\xi_{0}$.

The vector field $X_{t}$ generating $\varphi_{t}$ can be chosen in $\lim _{\varepsilon \rightarrow 0} \xi_{t} \cap \xi_{t+\varepsilon}$ at each time $t$.

Proof. The proof of this theorem can be found in many places but without much geometric explanations. So we now explain the picture behind it. The key is to be able to construct an isotopy pulling back $\xi_{t+\varepsilon}$ to $\xi_{t}$ for infinitesimally small $\varepsilon$. It means we will construct the generating vector field $X_{t}$ rather than $\varphi_{t}$ directly. The compactness assumption will guaranty that the flow of $X_{t}$ exists for all time. 
At any point $p$, if the plane $\xi_{t+\varepsilon}$ coincides with $\xi_{t}$ then we have nothing to do and set $X_{t}=0$. Otherwise, these two planes intersect transversely along a line $d_{t, \varepsilon}$. The natural way to bring $\xi_{t+\varepsilon}$ back to $\xi_{t}$ is to rotate it around $d_{t, \varepsilon}$. Since we know from the proof of Theorem 2 that the flow of Legendrian vector fields rotate the contact structure, we will choose $X_{t}$ in the line $d_{t}:=\lim _{\varepsilon \rightarrow 0} d_{t, \varepsilon}$, see Figure 1.9. Let us compute $d_{t, \varepsilon}$ :

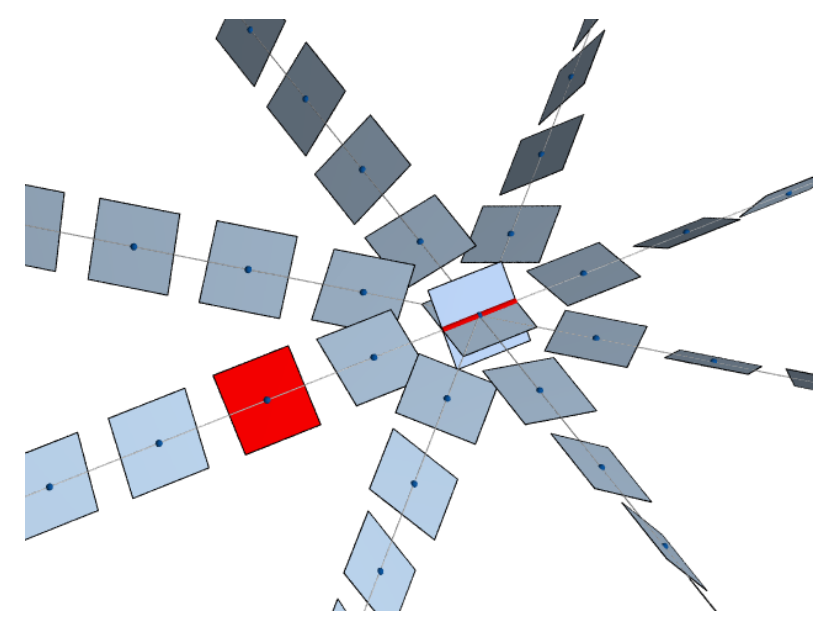

Figure 1.9: Proof of Gray's theorem

$$
d_{t, \varepsilon}=\left\{v \mid \alpha_{t+\varepsilon}(v)=\alpha_{t}(v)=0\right\}=\left\{v \in \xi_{t} \mid \frac{1}{\varepsilon}\left(\alpha_{t+\varepsilon}-\alpha_{t}\right)(v)=0\right\}
$$

which gives, as $\varepsilon$ goes to zero: $d_{t}=\xi_{t} \cap \operatorname{ker}\left(\dot{\alpha}_{t}\right)$.

The contact condition for $\alpha_{t}$ is equivalent to the fact that $\left(d \alpha_{t}\right)_{\mid \xi_{t}}$ is nondegenerate. So $X_{t}$ belongs to $\xi_{t} \cap \operatorname{ker}\left(\dot{\alpha}_{t}\right)$ if and only if it belongs to $\xi_{t}$ and $\iota_{X_{t}} d \alpha_{t}=f_{t} \dot{\alpha}_{t}$ on $\xi_{t}$ for some function $f_{t}$.

Moreover, we want $X_{t}$ to compensate the rotation expressed by $\dot{\alpha}_{t}$. A natural guess is then to pick the unique Legendrian vector field $X_{t}$ such that $\left(\iota_{X_{t}} d \alpha_{t}\right)_{\mid \xi_{t}}=-\left(\dot{\alpha}_{t}\right)_{\mid \xi_{t}}$.

We now have a precise candidate for $X_{t}$ and we can compute to prove that it does the job. Let $\varphi_{t}$ be the flow of $X_{t}$. Using Cartan's formula, we get:

$$
\begin{aligned}
\frac{d}{d t} \varphi_{t}^{*} \alpha_{t} & =\varphi_{t}^{*}\left(\dot{\alpha}_{t}+\mathcal{L}_{X_{t}} \alpha_{t}\right) \\
& =\varphi_{t}^{*}\left(\dot{\alpha}_{t}+\iota_{X_{t}} d \alpha_{t}\right) .
\end{aligned}
$$

By construction, the term in the parenthesis vanishes on $\xi_{t}$ so it is $\alpha_{t}$ multiplied by some function $\mu_{t}$ and we get:

$$
\frac{d}{d t} \varphi_{t}^{*} \alpha_{t}=\left(\mu_{t} \circ \varphi_{t}\right) \varphi_{t}^{*} \alpha_{t}
$$

So $\varphi_{t}^{*} \alpha_{t}$ stays on a line in the space of one forms. This line is obviously the line spanned by $\varphi_{0}^{*} \alpha_{0}=\alpha_{0}$ and we then have $\operatorname{ker} \varphi_{t}^{*} \alpha_{t}=\operatorname{ker} \alpha_{0}=\xi_{0}$ for all $t$. It is not hard to see that $X_{t}$ is the only Legendrian vector field which works. 
Some compactness assumption is indeed necessary in Gray's theorem. There are counter-examples on $\mathbb{R}^{2} \times \mathbb{S}^{1}$ discovered in Eli91.

Contact structures form an open set in the space of all plane fields. Gray's theorem proves that isotopy classes of contact structures on a closed manifold are actually connected components of this open set. In particular there are only finitely many isotopy classes of contact structures on a closed manifold.

The example of linear foliations on $T^{3}$ proves that Gray's theorem wouldn't hold for foliations.

\subsubsection{Libermann's theorem on contact Hamiltonians}

Contact transformations of a contact manifold $(V, \xi)$ are diffeomorphisms of $V$ which preserve $\xi$. The infinitesimal version of these are vector fields whose flow consists of contact transformations. They are called contact vector fields and are exactly those $X$ for which $\left(\mathcal{L}_{X} \alpha\right)_{\mid \xi}=0$ for any contact form $\alpha$ defining $\xi$. Note that this condition is weaker than $\mathcal{L}_{X} \alpha=0$ which would imply that the flow of $X$ preserves $\alpha$ and not only its kernel $\xi$.

In the proof of Gray's theorem, we saw that one can rotate a contact structure at will using the flow of a Legendrian vector field uniquely determined by the rotation we want to achieve. The same idea allows to prove that any vector field on a contact manifold can be transformed into a contact vector field by adding a uniquely determined Legendrian vector field. This is the geometric fact underlying the existence of so-called contact Hamiltonians.

Theorem 5 (Libermann Lib59). On a contact manifold $(V, \xi)$ the map which sends a contact vector field to its reduction modulo $\xi$ is an isomorphism from the space of contact vector fields to the space of sections of the normal bundle $T V / \xi$.

If we single out a contact form $\alpha$ then we get a trivialization $T V / \xi \rightarrow V \times \mathbb{R}$ given by $(x,[u]) \mapsto(x, \alpha(u))$. Sections of $T V / \xi$ can then be seen as functions on $V$ and the contact vector field $X_{f}$ associated to a function $f$ using the preceding theorem is called the Hamiltonian vector field coming from $\alpha$ and $f$. Libermann's theorem both implies existence of $X_{f}$ and the fact that it is the unique contact vector field satisfying $\alpha(X)=f$. The situation is analogous to the case of Hamiltonian vector fields in symplectic geometry but in the symplectic case there are symplectic vector fields that are not Hamiltonian. Note that the above interpretation when a contact form is fixed is what Libermann originally discussed and also the most common use of the word contact Hamiltonian.

Proof of Theorem 5. Let $X$ be any vector field on $V$. The theorem is equivalent to the assertion that there is a unique Legendrian vector field $X_{\xi}$ such that $X+X_{\xi}$ is contact. Using any contact form $\alpha$, we have equivalent reformulations:

$$
\begin{aligned}
X+X_{\xi} \text { is contact } & \Longleftrightarrow\left(\mathcal{L}_{X+X_{\xi}} \alpha\right)_{\mid \xi}=0 \\
& \Longleftrightarrow\left(\iota_{X+X_{\xi}} d \alpha+d\left(\iota_{X} \alpha\right)\right)_{\mid \xi}=0 \\
& \Longleftrightarrow\left(\iota_{X_{\xi}} d \alpha\right)_{\mid \xi}=-\left(\iota_{X} d \alpha+d\left(\iota_{X} \alpha\right)\right)_{\mid \xi}
\end{aligned}
$$

and the later condition defines uniquely $X_{\xi}$ because $d \alpha_{\mid \xi}$ is non-degenerate. 
Remark 6. A common use of contact Hamiltonians, and the only one we will need, is to cut-off or extend a contact vector field. For instance if $X$ is a contact vector field defined on an open set $U \subset V$ and $F$ is a closed subset of $V$ contained in $U$ then there is a contact vector field $\tilde{X}$ which vanishes outside $U$ and equals $X$ on $F$. If $L$ denotes the isomorphism of Theorem 5 and $\rho$ is a function with support in $U$ such that $\rho_{\mid F} \equiv 1$ then we can use $\tilde{X}=L^{-1}(\rho L(X))$. 


\section{Setting the goals: the tight vs overtwisted dichotomy}

After the local theory and before starting our study of convex surfaces, we need some motivation.

In Figure 1.7 showing $\operatorname{ker}\left(d z+r^{2} d \theta\right)$, the contact planes rotate along rays perpendicular to the $z$-axis but are never horizontal away from the $z$-axis. On the other extreme one can instead consider a contact structure which turns infinitely many times along these rays. A possible contact form for this is $\cos (r) d z+r \sin (r) d \theta$ which is horizontal for each $r$ such that $\sin (r)=0$, ie $r=k \pi$. Figure 1.10 shows what happens along $z=0$ and $r \leq \pi$. One sees a disk whose tangent space agrees with $\xi$ at the center and along the boundary.

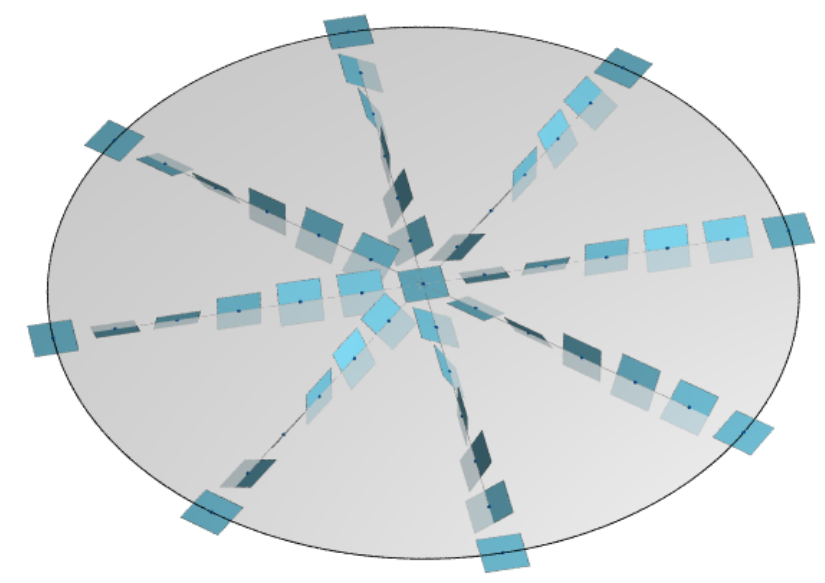

Figure 1.10: An overtwisted contact structure

Definition 7 (Eliashberg). A contact manifold is overtwisted if it contains an embedded disk along which the contact structure is as in Figure 1.10: the contact structure $\xi$ is tangent to the disk in the center and along the boundary and tangent to rays from the center to the boundary. A contact structure which is not overtwisted is called tight. 
It may look like this is the beginning of an infinite series of definitions where ones looks at disks $z=0, r \leq k \pi$ in the model above. But this would bring nothing new as can be seen from the following exercise.

Exercise. Prove that any neighborhood of an overtwisted disk in a contact manifold contains a whole copy of $\left(\mathbb{R}^{3}, \xi_{\text {От }}\right)$ where $\xi_{\text {От }}=\operatorname{ker}(\cos (r) d z+r \sin (r) d \theta)$.

The above exercise is pretty challenging at this stage but it can serve as a motivation for the technology at the beginning of the next chapter. And, most of all, it shows that not immediately seeing something in a contact manifold does not mean it is not there (recall also Figure 1.6). This begins to highlight the depth of the following two results whose proof is the main goal of these lecture notes.

Theorem 8 (Bennequin 1982 Ben83). The standard contact structures on $\mathbb{R}^{3}$ and $\mathbb{S}^{3}$ are tight.

Theorem 9 (Eliashberg 1992 Eli92]). All tight contact structures on $\mathbb{R}^{3}$ or $\mathbb{S}^{3}$ are isomorphic to the standard ones.

Bennequin's theorem shows in particular that the standard contact structure on $\mathbb{R}^{3}$ is not isomorphic to the overtwisted structure of Figure 1.10 . In order to put this in perspective, recall that Figures 1.4 and 1.5 show isomorphic contact structures. It may look like the difference between these is analogous to the difference between Figure 1.7 and 1.10. But Bennequin's theorem proves that the later two pictures are really different.

Eliashberg's theorem shows that tight contact structures on $\mathbb{S}^{3}$ are rare. By contrast, overtwisted contact structures abound. The Lutz-Martinet theorem, revisited by Eliashberg, says that, on a closed oriented manifold, any plane field is homotopic to an overtwisted contact structure Eli89. Recall that, because the Euler characteristic of a 3-manifold always vanishes, all such manifolds have plane fields and even more, there are always infinitely many homotopy classes of plane fields (for the classification of homotopy classes of plane fields one can refer to Gei08, Section 4.2]).

In CGH09], Colin, Giroux and Honda proved that only finitely many homotopy classes of planes fields on each manifold can contain tight contact structures. This is far beyond the scope of these lectures but see Theorem 30 for a weaker version due to Eliashberg Eli92. 


\section{Chapter 2}

\section{Convex surfaces}

The goal of this chapter is to explain the following crucial observation by Emmanuel Giroux in 1991:

If $S$ is a generic surface in a contact 3-manifold, all the information about the contact structure near $S$ is contained in an isotopy class of curves on $S$.

All this chapter except the last section comes from Giroux's PhD thesis Gir91, see also the webpage of Daniel Mathews for his translation of that paper into English.

\subsection{Characteristic foliations of surfaces}

After the local theory which explains what happens in neighborhoods of points in contact manifolds, we want to start the semi-local theory which deals with neighborhoods of surfaces.

The main tool will be characteristic foliations. The basic idea is to look at the singular foliation given on a surface $S$ by the line field $T S \cap \xi$, see Figure 2.1.

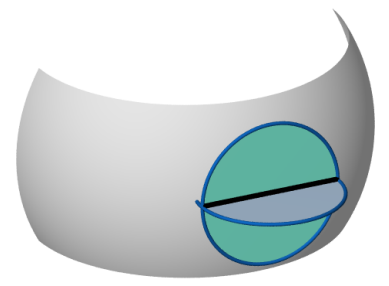

Figure 2.1: Characteristic foliation of a surface as the intersection between the tangent space and the contact plane.

In order to define precisely what is a line field with singularities, we see them as vector fields whose scale has been forgotten. It means they are equivalence 
classes of vector fields where $X \sim Y$ if there is a positive function $f$ such that $X=f Y$. A singularity is then a point where some, hence all, representative vanishes. Note that $f$ should be positive everywhere, including singularities.

One can think of a line as the kernel of a linear form rather than a subspace spanned by a vector. This prompts an equivalent definition as an equivalence class of 1 -forms where $\alpha \sim \beta$ if there is a positive function $f$ such that $\alpha=f \beta$.

To go from one point of view to the dual one, we can use an area form $\omega$ on the surface. The correspondence between vector fields and 1 -forms is then given by $X \mapsto \beta:=\iota_{X} \omega$. The singular foliations $[X]$ defined by $X$ and $[\beta]$ defined by $\beta$ are indeed geometrically the same since $X$ and $\beta$ vanish at the same points and elsewhere $X$ spans $\operatorname{ker} \beta$. In addition, one has the following commutative diagram which will be useful later.

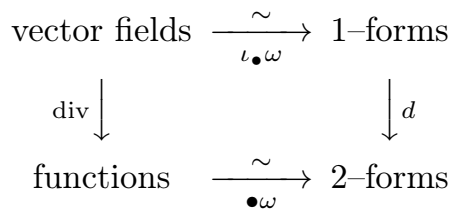

The left-hand side vertical arrow is the divergence map defined by the equality $\mathcal{L}_{X} \omega=(\operatorname{div} X) \omega$. So positive divergence means the flow of $X$ expands area while negative divergence means area contraction. Divergence is not well defined for a singular foliation because it depends on the representative vector field. However, at a singularity of a foliation, the sign of divergence is well defined because

$$
\mathcal{L}_{f X} \omega=d f \wedge \iota_{X} \omega+f(\operatorname{div} X) \omega
$$

so, at points where $X$ vanishes, $\operatorname{div} f X=f \operatorname{div} X$. The same kind of computation proves that this sign doesn't depend on the choice of the area form within a given orientation class.

Definition 10. Let $S$ be an oriented surface in a contact manifold $(M, \xi)$ with $\xi=\operatorname{ker} \alpha$, co-oriented by $\alpha$. The characteristic foliation $\xi S$ of $S$ is the equivalence class of the 1 -form $\iota^{*} \alpha$ induced by $\alpha$ on $S$.

In particular, singularities of the characteristic foliation $\xi S$ are points where $\xi=T S$ (maybe with reversed orientation). At those points $d \iota^{*} \alpha=d \alpha_{\mid \xi}$ is non-degenerate so the above commutative diagram proves that singularities of characteristic foliations have non-zero divergence.

Examples Figures 2.2, 2.3 and 2.4 show examples of characteristic foliations.

\subsubsection{Leaves of characteristics foliations}

The leaves (or orbits) of a singular foliation are the integral curves of any vector field representing it. The intuitive notion of a singular foliation is rather the data of leaves than an equivalence class of vector fields. In contact geometry, this discrepancy does not generate any confusion thanks to the following lemma. It is a rather technical point but we discuss it here anyway because it doesn't appear to be published anywhere else, although it is mentioned in Gir00 page $629]$. 


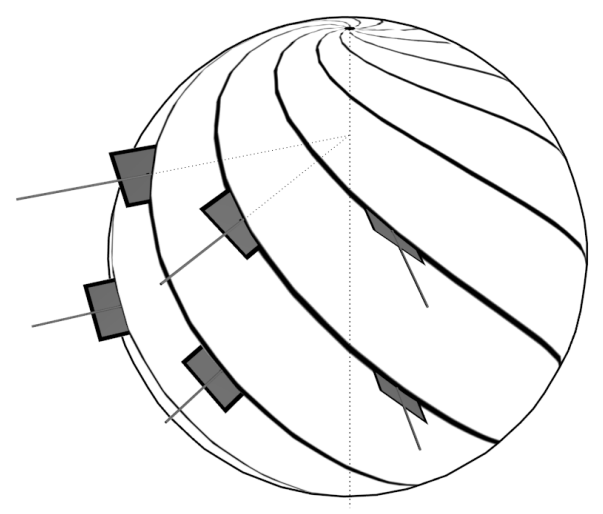

Figure 2.2: Characteristic foliation of Euclidean spheres around the origin in $\mathbb{R}^{3}$ equipped with the canonical contact structure $\xi=\operatorname{ker}\left(d z+r^{2} d \theta\right)$. There are singular points at the intersection with the $z$-axis and all regular leaves go from a singularity to the other one.

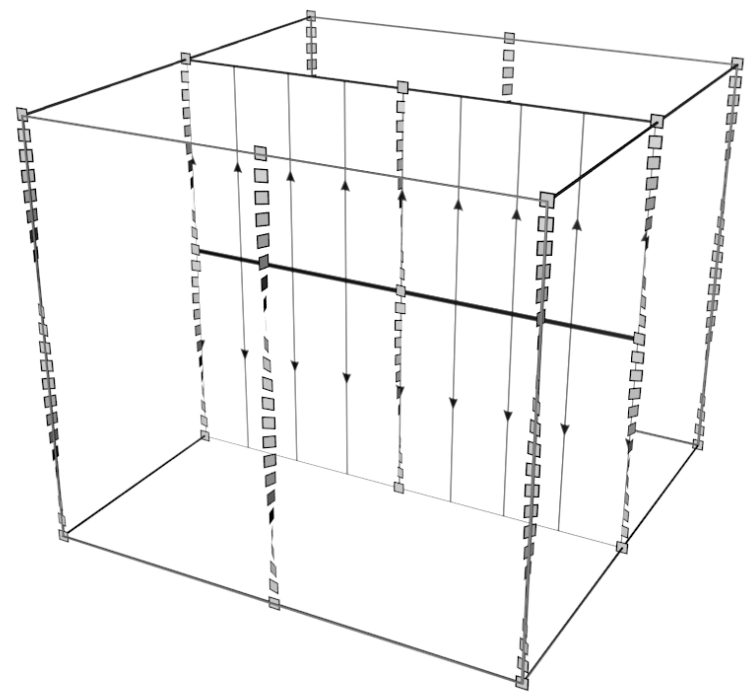

Figure 2.3: Characteristic foliation of a torus $\{x=$ constant $\}$ in $T^{3}$ equipped with its canonical contact structure $\xi=\operatorname{ker}(\cos (z) d x-\sin (z) d y)$. One can see two circles made entirely of singularities where $\sin (z)=0$, one appear in the middle of the picture and the other one can be seen both at bottom and at top. 


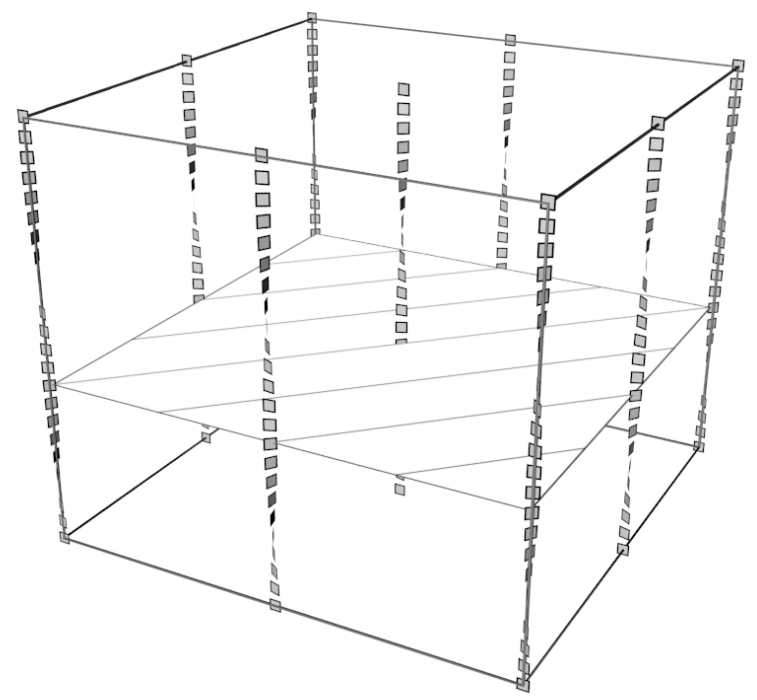

Figure 2.4: Characteristic foliation of a torus $\{z=$ constant $\}$ in $T^{3}$ equipped with its canonical contact structure $\xi=\operatorname{ker}(\cos (z) d x-\sin (z) d y)$.

Lemma 11 (Giroux). If two singular foliations on a surface have the same leaves and if their singularities have non-zero divergence then they are equal.

The following proof can be safely skipped on first reading.

Proof. The statement is clear away from singularities and a partition of unity argument brings it down to a purely local statement. So we focus on a neighborhood of a singularity (which may be non-isolated though).

Let $Y$ and $Y^{\prime}$ be vector fields on $\mathbb{R}^{2}$ which vanish at the origin and have the same orbits.

$$
Y=f \partial_{x}+g \partial_{y} \quad \text { et } \quad Y^{\prime}=f^{\prime} \partial_{x}+g^{\prime} \partial_{y} .
$$

We will compute divergence using the Euclidean area form $\omega=d x \wedge d y$ (we know the sign of divergence of singular points does not depend on this choice). So $\operatorname{div} Y=\partial_{x} f+\partial_{y} g$. All the following assertions will be true in a neighborhood of the origin that will shrink only finitely many times. $\operatorname{Since} \operatorname{div}(Y)$ is non-zero, we can use a linear coordinate change to ensure that $\partial_{x} f$ doesn't vanish. The implicit function theorem then gives new coordinates such that $f(x, y)=x$. Because

$$
f^{\prime}(x, y)=f^{\prime}(0, y)+x \int_{0}^{1} \partial_{x} f^{\prime}(t x, y) d t
$$

we can write $f^{\prime}=x u(x, y)+v(y)$. Along the curve $\{x=0\}$, the vector field $Y$ is vertical (or zero) so the same is true for $Y^{\prime}$. Hence $f^{\prime}$ also vanishes along this curve and $v$ is identically zero. The condition that $Y$ and $Y^{\prime}$ are either 
simultaneously zero or colinear is then:

$$
\left|\begin{array}{cc}
x & x u \\
g & g^{\prime}
\end{array}\right|=0
$$

which gives $g^{\prime}=u g$ where $x$ is non-zero hence everywhere by continuity. One then gets $Y^{\prime}=u Y$. In particular $\operatorname{div} Y^{\prime}=u \operatorname{div} Y+d u \wedge\left(\iota_{Y} d x \wedge d y\right)$. Away from zeros of $Y$ and $Y^{\prime}, u$ is positive because $Y$ and $Y^{\prime}$ have the same leaves. At a common zero, $\operatorname{div} Y^{\prime}=u \operatorname{div} Y$ and, because singularities of $Y^{\prime}$ have non-zero divergence, the function $u$ doesn't vanish. Hence it is positive everywhere (note that $Y$ and $Y^{\prime}$ can't be everywhere zero).

\subsection{Neighborhoods of surfaces}

Any orientable surface $S$ in an orientable 3-manifold has a neighborhood diffeomorphic to $S \times \mathbb{R}$ (use the flow of a vector field transverse to $S$ ). We will always denote by $t$ the coordinate on $\mathbb{R}$ and by $S_{t}$ the surface $S \times\{t\}$ for a fixed $t$. From now on, we will assume that $S$ is oriented and orient $S \times \mathbb{R}$ as a product.

Any plane field $\xi$ defined near $S$ has then an equation $\alpha=u_{t} d t+\beta_{t}$ where $u_{t}$ is a family of functions on $S$ and $\beta_{t}$ is a family of 1 -forms on $S$. Note that the characteristic foliation of $S_{t}$ is the equivalence class of $\beta_{t}$ since the latter is the 1-form induced by $\alpha$ on $S_{t}$.

The contact condition for $\xi$ (with respect to the product orientation) is equivalent to

$$
u_{t} d \beta_{t}+\beta_{t} \wedge\left(d u_{t}-\dot{\beta}_{t}\right)>0
$$

where $\dot{\beta}_{t}$ denotes $\frac{\partial \beta_{t}}{\partial t}$. This condition is a non-linear partial differential relation which is not so simple. The main thrust of the following discussion will be to simplify it by fixing some of the terms.

\section{Reconstruction lemmas}

The easiest case is to fix the whole family $\beta_{t}$. In this case the contact condition (®) is only about the family $u_{t}$ and becomes convex. In particular the space of solutions $u_{t}$ is connected and we get:

Lemma 12 (Global reconstruction). If $\xi$ and $\xi^{\prime}$ are positive contact structures on $S \times \mathbb{R}$ such that $\xi S_{t}=\xi^{\prime} S_{t}$ for all $t$ then $\xi$ and $\xi^{\prime}$ are isotopic.

We give a detailed proof since it is a model of several later proofs.

Proof. There are equations $u_{t} d t+\beta_{t}$ and $u_{t}^{\prime} d t+\beta_{t}^{\prime}$ of $\xi$ and $\xi^{\prime}$. The hypothesis of the lemma is that $\beta_{t}^{\prime}=f_{t} \beta_{t}$ for some family of positive functions $f_{t}$ on $S$. So another equation for $\xi^{\prime}$ is $u_{t}^{\prime} / f_{t} d t+\beta_{t}$. We have two solutions $u_{t}$ and $u_{t}^{\prime} / f_{t}$ of the contact condition, Equation ( $夫$, with $\beta_{t}$ fixed. Since this condition is convex, the space of its solutions is connected so we can find a family of solution $\left(u_{t}^{s}\right)_{s \in[0,1]}$ relating them (a linear interpolation will do the job). This family corresponds to a family of contact structures $\xi_{s}=\operatorname{ker}\left(u_{t}^{s} d t+\beta_{t}\right)$ which Gray's theorem (Theorem 44) converts to an isotopy of contact structures ${ }^{1}$

\footnotetext{
${ }^{1}$ One may worry about the fact that $S \times \mathbb{R}$ is non-compact but here the vector field constructed during the proof of this theorem is tangent to $S_{t}$ which is compact for all $t$ hence its flow is well defined for all times
} 
Our discussion of Gray's theorem actually tells us more about what is going on. Recall the vector field generating the isotopy at time $s$ can be chosen in the intersections of $\xi_{s}$ and $\xi_{s+\varepsilon}$. So we see the isotopy is stationary at each singular point of the characteristic foliations $\left[\beta_{t}\right]$. At all other points it is tangent to the characteristic foliation and its flow makes the contact structures we want to relate to rotate toward each other, see Figure 2.5 .

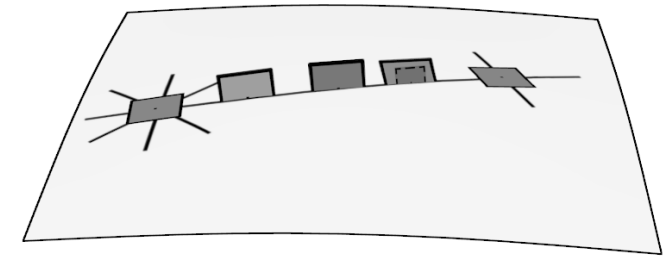

Figure 2.5: Reconstruction lemmas. We have two contact structures printing the same characteristic foliation on a surface. One of them is drawn along an arc going from a singularity to another. The second one appears only at one point with dotted outline. At this point the isotopy constructed in the proof is tangent to the arc to make the contact structure rotate.

If instead of fixing the whole family $\beta_{t}$ we fix only $\beta_{0}$ then we get the following lemma.

Lemma 13 (Local reconstruction). If $\xi$ and $\xi^{\prime}$ are positive contact structures which prints the same characteristic foliation on a compact embedded surface $S$ then there is a neighborhood of $S$ on which $\xi$ and $\xi^{\prime}$ are isotopic (by an isotopy globally preserving $S$ ).

Proof. The contact condition along $S_{0}$ becomes a convex condition on $u_{0}$ and $\dot{\beta}_{0}$. Again we can find a path of plane fields which, along $S$, are contact structures interpolating between $\xi$ and $\xi^{\prime}$. Because the contact condition is open, they will stay contact structures near $S$ and we can use Gray's theorem again.

Exercise. Prove that the two preceding lemmas are false for foliations.

We can now return to the challenging exercise of Chapter 1 with much better chances of success. Recall that $\xi_{\text {От }}=\operatorname{ker}(\cos (r) d z+r \sin (r) d \theta)$.

Exercise. Use the local reconstruction lemma to prove that any neighborhood of an overtwisted disk in a contact manifold contains a copy of $\left(\mathbb{R}^{3}, \xi_{\text {от }}\right)$. Hint: try to understand the characteristic foliation of the surface of Figure 2.6.

As illustrated by the previous exercise, the reconstruction lemmas are already quite useful by themselves. But the characteristic foliation is still a huge data and it is very sensitive to perturbations of the contact structure or the surface. This will be clear from the discussion of genericity of convex surfaces and of the realisation lemma below. 


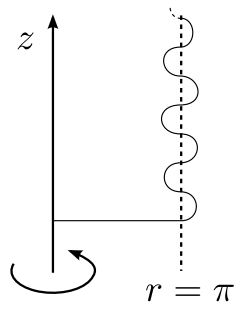

Figure 2.6: Rotating the wavy curve around the $z$-axis in $\left(\mathbb{R}^{3}, \xi_{\text {от }}\right)$ gives a plane having a characteristic foliation diffeomorphic to that of $\{z=0\}$. Note that the curve is horizontal at each intersection with the $\{r=\pi\}$ axis.

\subsubsection{Convex surfaces}

\section{Homogeneous neighborhoods}

The next step in our quest to simplify the contact condition ( $\star$ ) seems to be fixing $u_{t}$ instead of $\beta_{t}$. But this still gives a non-linear equation on the family $\beta_{t}$ if $\dot{\beta}_{t}$ is not zero. So we assume that $\beta_{t}$ does not depend on $t: \beta_{t}=\beta$. In particular the families $\left(u_{0}, \beta\right)$ and $\left(u_{t}, \beta\right)$ both give contact structures with the same characteristic foliation $[\beta]$ on each $S_{t}$. Hence the global reconstruction Lemma tells us these contact structures are isotopic. So we now assume that $u_{t}$ is also independent of $t$.

In this situation, the contact structure itself becomes invariant under $\mathbb{R}$ translations, one says that $\partial_{t}$ is a contact vector field. Note that this vector field is transverse to all surfaces $S_{t}$. Conversely if a contact vector field is transverse to a surface then it can be cut-off away from the surface using Remark 6 and then its flow defines a tubular neighborhood $S \times \mathbb{R}$ with a $t$-invariant contact structure.

Definition 14 (Giroux Gir91). A surface $S$ in a contact 3-manifold $(M, \xi)$ is $\xi$-convex if it is transverse to a contact vector field or, equivalently, if it has a so called homogeneous neighborhood: a tubular neighborhood $S \times \mathbb{R}$ where the restriction of $\xi$ is $\mathbb{R}$-invariant.

Example 15. In $T^{3}$ with its canonical contact structure, all tori $\{x=$ constant $\}$ as in Figure 2.3 are $\xi$-convex since they are transverse to the contact vector field $\partial_{x}$.

Example 16. In $\left(\mathbb{R}^{3}, \operatorname{ker}\left(d z+r^{2} d \theta\right)\right)$, any Euclidean sphere around the origin is $\xi$-convex since they are transverse to the contact vector field $x \partial_{x}+y \partial_{y}+2 z \partial_{z}$.

In the convex case, the contact condition becomes:

$$
u d \beta+\beta \wedge d u>0
$$

Using some area form $\omega$ and Equation 2.1), one can rephrase it in terms of the vector field $Y \omega$-dual to $\beta$ as:

$$
u \operatorname{div}_{\omega} Y-d u(Y)>0
$$


Analogously to the previous section we see that, $u$ being fixed, the space of solutions $\beta$ to (†) is contractible, this was our stated goal when we asked $\beta_{t}$ to be independent of $t$. The miracle is that it essentially stays true if one fixes only the zero set $\Gamma$ of $u$. Indeed, away from $\Gamma$, we can divide our contact form $u d t+\beta$ by $|u|$ to replace it by $\operatorname{ker}\left( \pm d t+\beta^{\prime}\right)$ where $\beta^{\prime}=\frac{1}{|u|} \beta$. The condition (†) for $\left( \pm 1, \beta^{\prime}\right)$ is simply $\pm d \beta^{\prime}>0$ which is not only convex, it does not depend on $u$ ! Of course this discussion needs some precise definitions which are provided below but the first miracle has already happened: near a $\xi$-convex surface $S$, all the information about $\xi$ is contained in $\Gamma$. It remains to see that such surfaces are generic, the second miracle.

\section{Dividing sets}

Let us take a look at $\Gamma=\{u=0\}$. Along $\Gamma$, the contact condition $\left(\sqrt{t^{\prime}}\right)$ reads $-d u(Y)>0$. So $\Gamma$ is a regular level set of $u$. Hence it is a one-dimensional submanifold without boundary, ie a collection of disjoint simple closed curves in $S$. Such collections will be referred to as multi-curves.

The condition $-d u(Y)>0$ also implies that $\Gamma$ is transverse to $\xi S$. More precisely, $Y$ goes from $S_{+}=\{u>0\}$ to $S_{-}=\{u<0\}$ along $\Gamma$ and the picture near $\Gamma$ is always as in Figure 2.7. In the following discussion we will use several time the fact that this picture is very simple and controlled to be less precise about what happens near $\Gamma$.

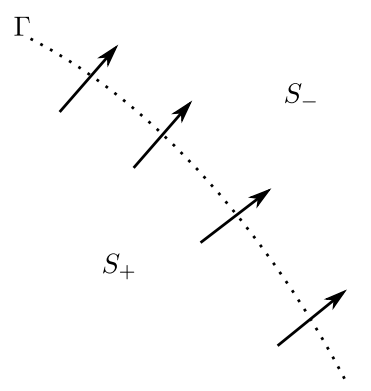

Figure 2.7: Characteristic foliation near the dividing set $\Gamma$

The last remarkable property of the decomposition of $S$ in $S_{+}$and $S_{-}$is $Y$ expands some area form in $S_{+}$and contracts it in $S_{-}$. Indeed, if one sets $\Omega=\frac{1}{|u|} \omega$ on $S \backslash \Gamma$ then $\operatorname{div}_{\Omega} Y= \pm \frac{1}{u^{2}}$ on $S_{ \pm}$. One can actually modify $\Omega$ near $\Gamma$ so that $\operatorname{div}_{\Omega} Y$ is positive on $S_{+}$, negative on $S_{-}$and vanishes along $\Gamma$.

Definition 17. A singular foliation $\mathscr{F}$ of a surface $S$ is divided by an (embedded) multi-curve $\Gamma$ if there is some area form $\Omega$ on $S$ and a vector field $Y$ directing $\mathscr{F}$ such that:

- the divergence of $Y$ does not vanish outside $\Gamma$-we set

$$
S_{ \pm}=\left\{p \in S ; \pm \operatorname{div}_{\Omega} Y(p)>0\right\}
$$

- the vector field $Y$ goes transversely out of $S_{+}$and into $S_{-}$along $\Gamma$. 
What we proved above is that the characteristic foliation of a $\xi$-convex surface is divided by some multi-curve. Using the local reconstruction lemma (Lemma 13, one can prove the converse to get:

Proposition 18. A surface $S$ is $\xi$-convex if and only if $\xi S$ is divided.

Proof. We assume that $\xi S$ is divided by some multi-curve $\Gamma$. According to the local reconstruction lemma, we only need to prove that there is a contact structure $\xi^{\prime}$ defined near $S$ such that $S$ is $\xi^{\prime}$-convex and $\xi^{\prime} S=\xi S$. We set $\beta=\iota_{Y} \Omega$. In particular $\xi S=[\beta]$. On $S \backslash \Gamma, \xi^{\prime}=\operatorname{ker} \pm d t+\beta$ is a contact structure which also prints $[\beta]$ on $S \backslash \Gamma$ and one can check that there is no problem to extend it along $\Gamma$.

Note that the dividing set is not unique for a given foliation. If $X$ is a contact vector field transverse to the surface $S$ then the considerations above prove that $\Gamma_{X}:=\{s \in S ; X(s) \in \xi\}$ is a dividing set for $S$.

However, if one fixes $\beta$ in the contact condition (†), it becomes convex in $u$, hence the space of solutions $u$ is connected. This implies that the space of multi-curves dividing a given foliation is connected (in fact contractible).

Examples In the case of spheres of example 16, the dividing set corresponding to the given vector field is the equator $\{z=0\}$.

In the torus case of Figure 2.3, the dividing set coming from $\partial_{x}$ is defined by $\cos (z)=0$ so it is made of two circles sitting between the singularity circles defined by $\sin (z)=0$, see Figure 2.8 .

\section{The realization lemma}

We are now ready to make precise the fact that the dividing set contains all the information about the contact structure near a convex surface.

Lemma 19 (Realization Lemma). Let $S$ be a $\xi$-convex surface divided by some multi-curve $\Gamma$. For any singular foliation $\mathscr{F}$ divided by $\Gamma$, there is an isotopy $\delta_{t}$ with support in an arbitrarily small neighborhood of $S$ and such that $\xi^{\prime}=\delta_{1}^{*} \xi$ satisfies $\xi^{\prime} S=\mathscr{F}$. Equivalently, one has $\xi \delta_{1}(S)=\delta_{1}(\mathscr{F})$.

So any singular foliation divided by $\Gamma$ is printed on $S$ by some contact structure isotopic to $\xi$ or, equivalently, it can be realized as the characteristic foliation of a surface isotopic to $S$.

The proof of this very important lemma has already been essentially explained right after stating condition (†). It follows from the fact that $\pm d \beta>0$ is a convex condition and Gray's theorem as in the reconstruction lemmas.

This lemma is often called Giroux's flexibility theorem but one can argue that it is rather a rigidity result since all the information can be stored into a tiny combinatorial data: the isotopy class of the dividing set.

Example Consider the convex torus of Figure 2.3. Its characteristic foliation is highly non generic since it has two circles of singularities. Yet it is divided by two circles parallel to the singularity circles. Figure 2.9 shows a generic foliation divided by the same curves but where singular circles have been replaced by regular closed leaves. 


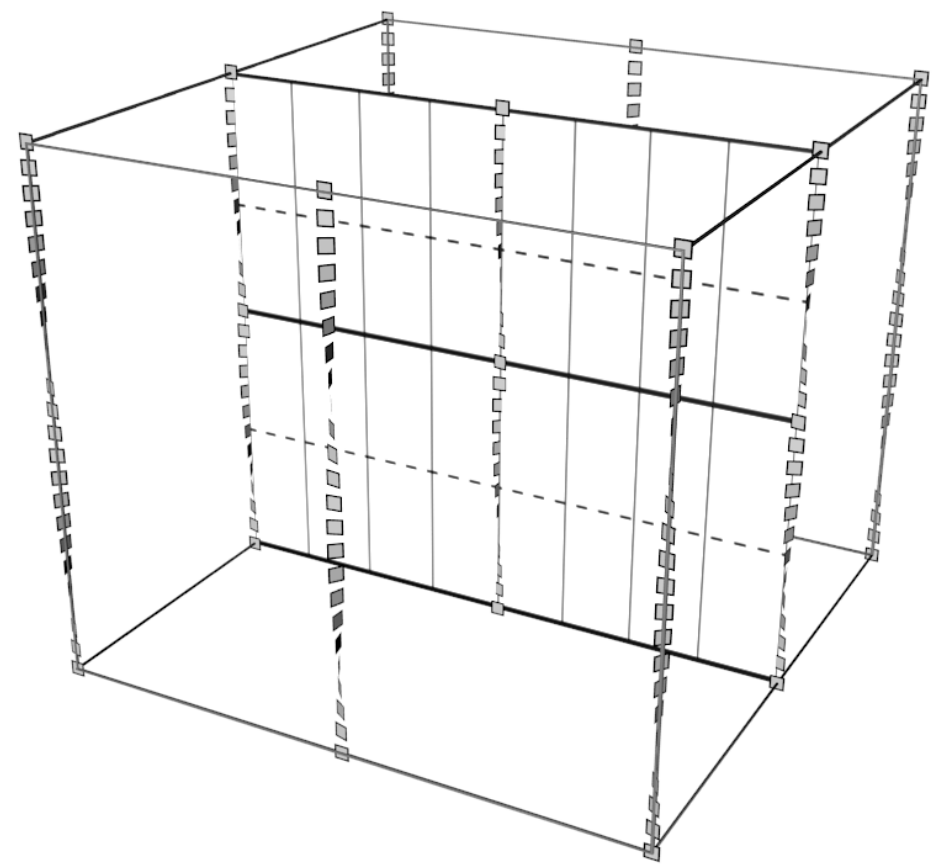

Figure 2.8: A dividing set for the torus of Figure 2.3 (dashed on the picture)

The realization lemma implies that the surface of Figure 2.3 is isotopic to a surface which has Figure 2.9 as its characteristic foliation. Figure 2.10 shows this surface explicitly.

The transition between these foliations play an important role in the classification of tight contact structures on the product of a torus and an interval, see [Gir00, Section 1.F]

In order to use the power of the realization lemma, we need to prove that $\xi$-convex surfaces exist in abundance. We will first discuss some obstructions to $\xi$-convexity then prove genericity of $\xi$-convex surfaces.

\subsubsection{Obstructions to convexity}

\section{Degenerate closed leaves}

The most obvious obstruction to $\xi$-convexity for a closed surface $S$ is when $\xi S$ is defined by some $\beta$ with $d \beta=0$, as in Figure 2.4 because then the contact condition (If) becomes $\beta \wedge d u>0$ which implies that $u$ has no critical point.

Surfaces with such characteristic foliations are called pre-Lagrangian.

They are either tori or Klein bottles and play an important role in some later part of the theory.

This obstruction idea can be extended remarking that it does not need the whole of $S$, it can be applied along a closed leaf $L$ of $\xi S$. This is easier to see in 


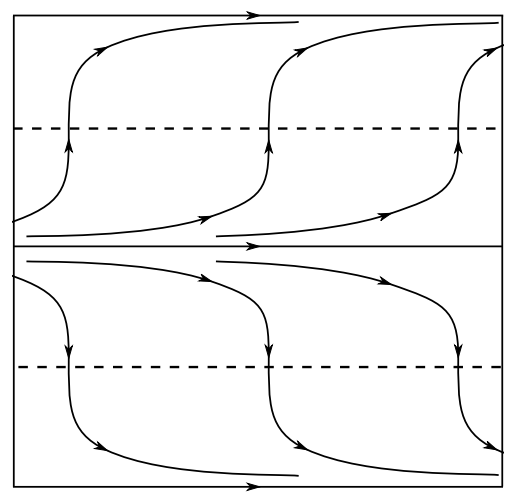

Figure 2.9: A generic foliation of the torus divided by two curves

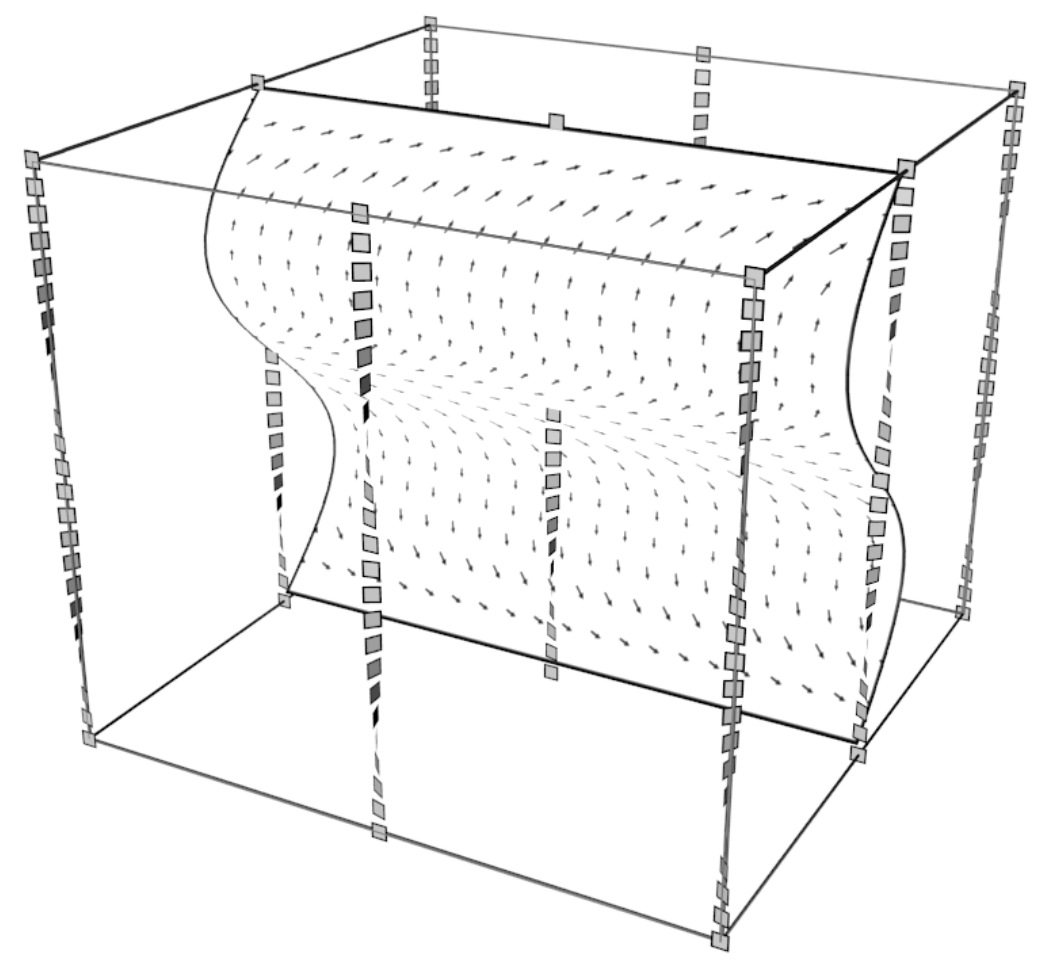

Figure 2.10: A realization of Figure 2.9 as a deformation of the torus of Figure 2.3 
the dual picture of equation $\left(\mathrm{t}^{\prime}\right)$. Indeed, if $\operatorname{div}_{\omega}(Y)$ vanishes along $L$, condition $\left(\dagger^{\prime}\right)$ says that $-u_{\mid L}^{\prime}>0$ whereas the restriction $u_{\mid L}$ necessarily has some critical point.

Definition 20. A closed leaf $L$ of a singular foliation is degenerate if there is a 1 -form $\beta$ defining the foliation near $L$ and whose differential $d \beta$ vanishes along $L$. A non-degenerate leaf is called repelling (resp attracting) if there is some $\beta$ such that $d \beta$ is positive (resp negative) along $L$.

The definition above is convenient for our purposes but one should keep in mind that it is equivalent to the more geometrical definition through Poincaré's first return map $\pi$ on a transverse curve $c$, see Figure 2.11. A closed leaf is degenerate if $\pi^{\prime}(0)=1$. A non-degenerate closed leaf is attracting if $\pi^{\prime}(0)<1$ and repelling if $\pi^{\prime}(0)>1$.

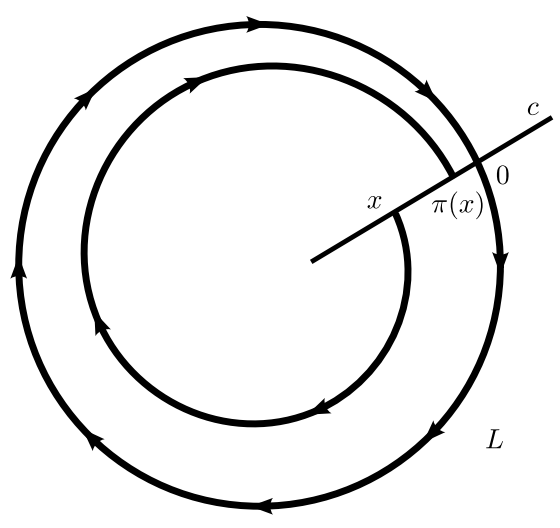

Figure 2.11: Poincaré's first return map $\pi$ on a transversal $c$ to a closed leaf $L$.

The discussion preceding the definition proves that if $S$ is $\xi$-convex then $\xi S$ has no degenerate closed leaves.

Remark 21. Suppose now that $S$ is indeed $\xi$-convex and $L$ is a (non-degenerate) closed leaf of $\xi S$. Let $\Gamma$ be a dividing set for $\xi S$. Because $\xi S$ is transverse to $\Gamma$ and always goes out of $S_{+}$and into $S_{-}, L$ cannot meet $\Gamma$. Because $L$ is compact, the restriction of $u$ to $L$ has at least one critical point. At this point, the contact condition gives $u d \beta>0$. So repelling orbits are in $S_{+}$and attracting orbits are in $S_{-}$.

\section{Retrograde connections}

Recall from Section 2.1 that the contact condition ensures that all singularities of characteristic foliations have non-zero divergence and hence have non-zero sign. Singularities of $\xi S$ correspond to points where $S$ is tangent to $\xi$ and they are positive or negative depending on whether the orientation of $\xi$ and $S$ match or not.

In generic characteristic foliations one sees only two topological types of singularities: nodes and saddles. If one considers generic families of characteristic foliations then saddle-nodes may appear, see Figure 2.14. Since the sign of 


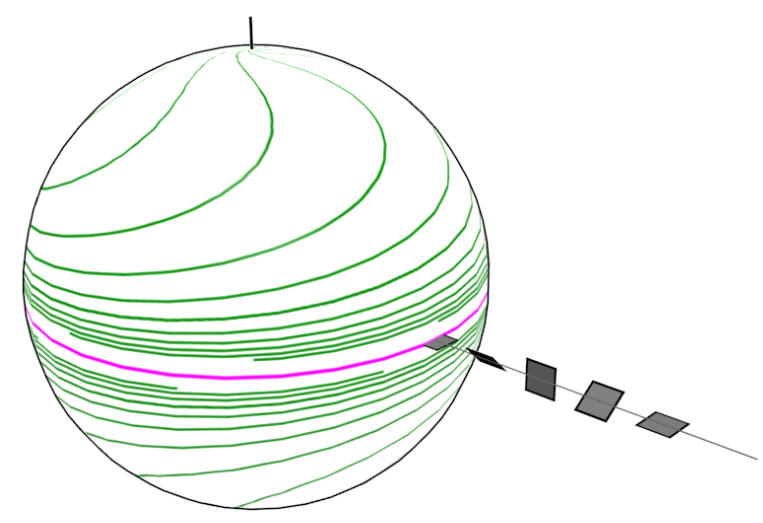

Figure 2.12: A sphere or radius $\pi$ in the overtwisted $\mathbb{R}^{3}$. The equator is a degenerate closed leaf. Note how leaves spiral a lot more around a degenerate leaf than around a non-degenerate.

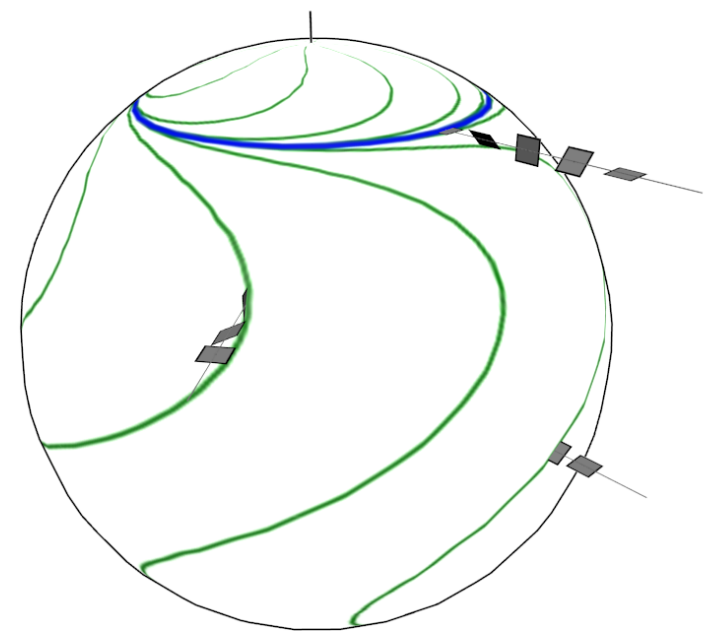

Figure 2.13: A sphere or radius slightly less than $2 \pi$ in the overtwisted $\mathbb{R}^{3}$. The intersection with the cylinder $\{r=\pi\}$ consists of two non-degenerate closed leaves (one of them is not visible in the picture). 


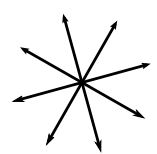

node

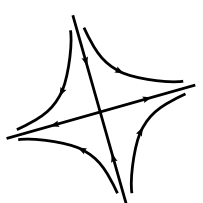

saddle

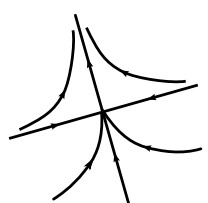

saddle-node

Figure 2.14: Generic singularities of characteristic foliations

singularities corresponds to their divergence, positive nodes are always sources while negative nodes are always sinks. The sign of saddles cannot be read from topological pictures only.

Let $S$ be a $\xi$-convex surface so that $\xi=\operatorname{ker}(u d t+\beta)$ near $S$. We begin by a remark analogous to Remark 21. At any singular point $p$ of $\xi S$, the contact condition (†) give $u d \beta(p)>0$. So singularities are positive in $S_{+}$and negative in $S_{-}$.

Suppose now that $p$ and $q$ are two singular points of $\xi S$ with opposite signs and there is a regular leaf $L$ of $\xi S$ going from $p$ to $q$. Because $L$ has to be transverse to $\Gamma$ and go from $S_{+}$to $S_{-}$, the above discussion proves that $p$ is positive and $q$ is negative.

Definition 22. In the characteristic foliation of a surface, a retrograde connection is a leaf which goes from a negative singularity to a positive one.

The discussion above proves that $\xi$-convex surfaces have no retrograde connections. Note that retrograde connections cannot involve nodes since the sign of nodes determine the local orientations of the foliation.

Leaves of characteristic foliations between two singularities of opposite signs are always arcs tangent to the contact structure along which the contact structure rotates half a turn compared to the surface. What makes retrograde connections special is that the direction of rotation is opposite to the one around Legendrian foliations.

Example 23 (Gir00, Example 3.41]). In $\mathbb{R}^{2} \times \mathbb{S}^{1}$ with contact structure $\xi=$ $\operatorname{ker}(\cos (2 \pi z) d x-\sin (2 \pi z) d y)$, we consider the family of transformations

$$
\varphi_{t}((x, y), z)=\left(R_{-4 \pi t}(x, y), z+t\right)
$$

where $R_{\theta}$ denotes the rotation of angle $\theta$ around the origin of $\mathbb{R}^{2}$. The orbit of a circle in $\mathbb{R}^{2}$ passing through the origin sweeps a torus $S$ whose characteristic foliation has two retrograde saddle connections along the z-axis, see Figure 2.15. Indeed, along this axis, the tangent plane TS turns in the same direction as $\xi$ but twice as fast. It means that, seen from $T S, \xi$ rotates one turn in the opposite direction. See Figure 2.16 for a better view of the characteristic foliation. 


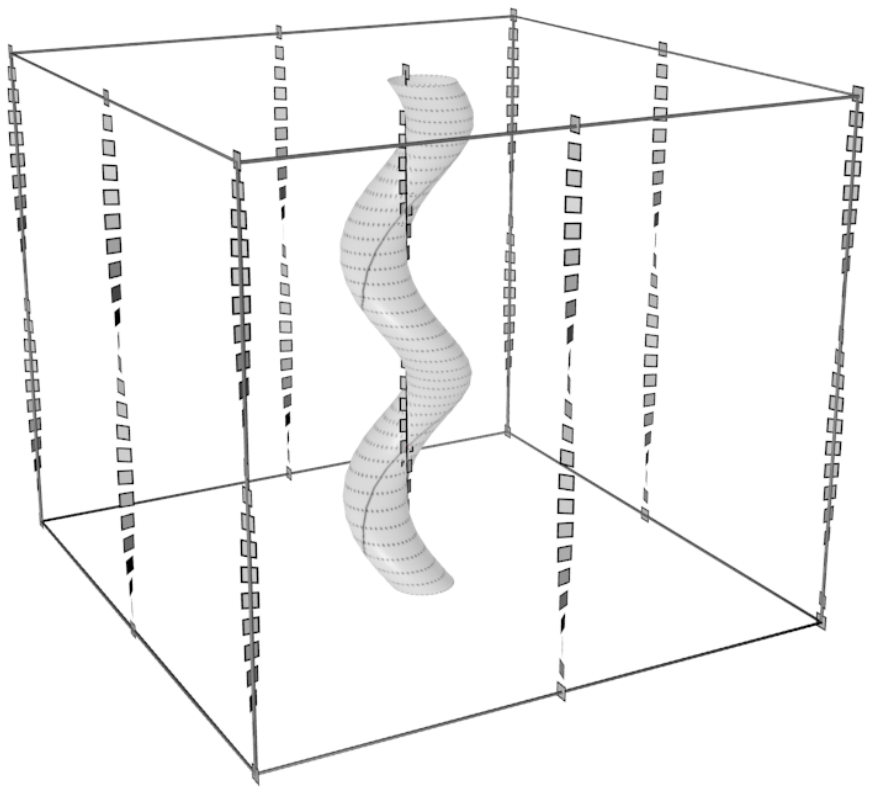

Figure 2.15: A torus having a retrograde saddle connection

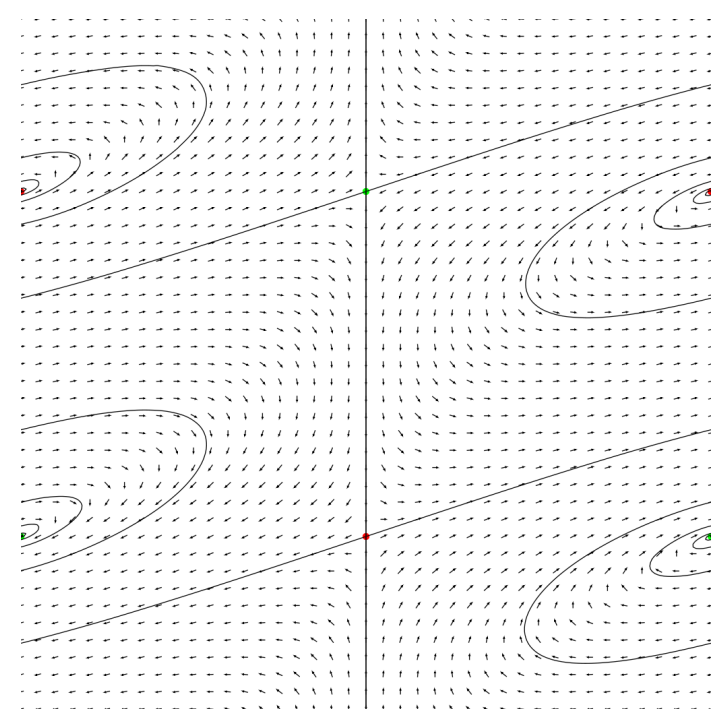

Figure 2.16: A (double) saddle connection on the torus of Figure 2.15 after top/bottom and left/right are glued. The top saddle is negative, the bottom one positive. The top node is positive, the bottom one negative. The curves drawn are all the separatrices of the saddles. 


\subsubsection{Genericity of convex surfaces}

We are now ready to use generic properties of vector fields on surfaces to prove that any surface in a contact manifold can be perturbed to a $\xi$-convex one. See Figures 2.17 and 2.18 for an example and [Gir01, Proof of Proposition 2.10] for more examples of the same kind.

Proposition 24. Any closed surface in a contact 3-manifold $(M, \xi)$ is $C^{\infty}$ close to a $\xi$-convex surface.

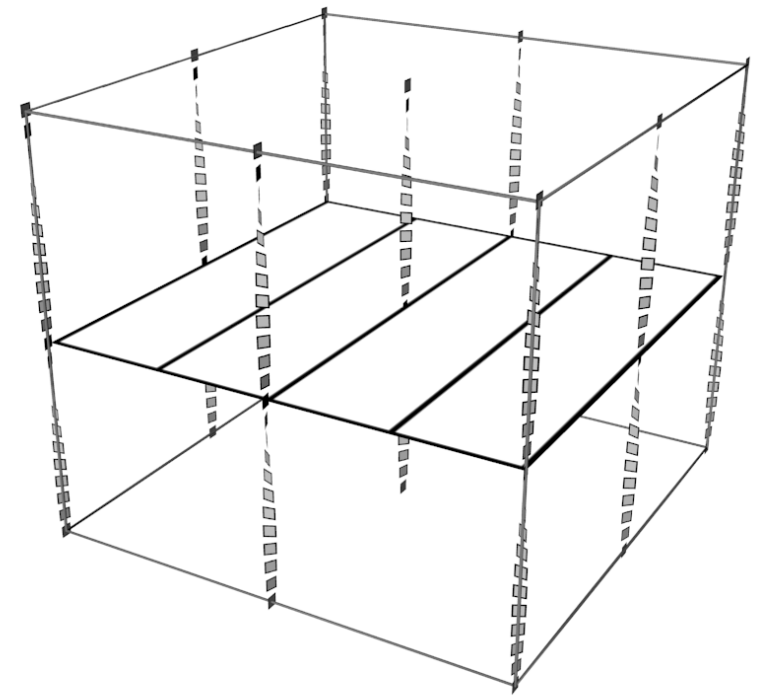

Figure 2.17: A non-convex torus

Genericity of $\xi$-convex surfaces is a small dimensional phenomenon, it does not hold for hypersurfaces in higher dimensions Mor11. In dimension 3, $\xi_{-}$ convexity is a degenerate notion, much like ordinary convexity in real dimension 1 and pseudo-convexity in complex dimension 1 .

We first prove that any foliation sufficiently close to a characteristic foliation $\xi_{0} S$ is the characteristic foliation $\xi S$ coming from some $\xi$ isotopic to $\xi_{0}$. Equivalently it means it is the characteristic foliation printed by $\xi_{0}$ on some surface isotopic to $S$. Let $C$ be the connected component of the space of contact structures which contains $\xi_{0}$. The first point is that the map which maps $\xi$ in $C$ to the characteristic foliation $\xi S$ is open. The second point is that Gray's theorem imply that all $\xi$ in $C$ are isotopic to $\xi_{0}$.

So the genericity of $\xi$-convex surfaces will follow from the one of divided foliations. Essentially we will see that the obstructions to the existence of a dividing set discussed above are the only ones provided that no non-trivial recurrence appear. The precise requirement is expressed in the following definition.

Definition 25. A singular foliation on a closed surface satisfies the PoincaréBendixson property if the limit set of any half orbit is either a singularity or a 


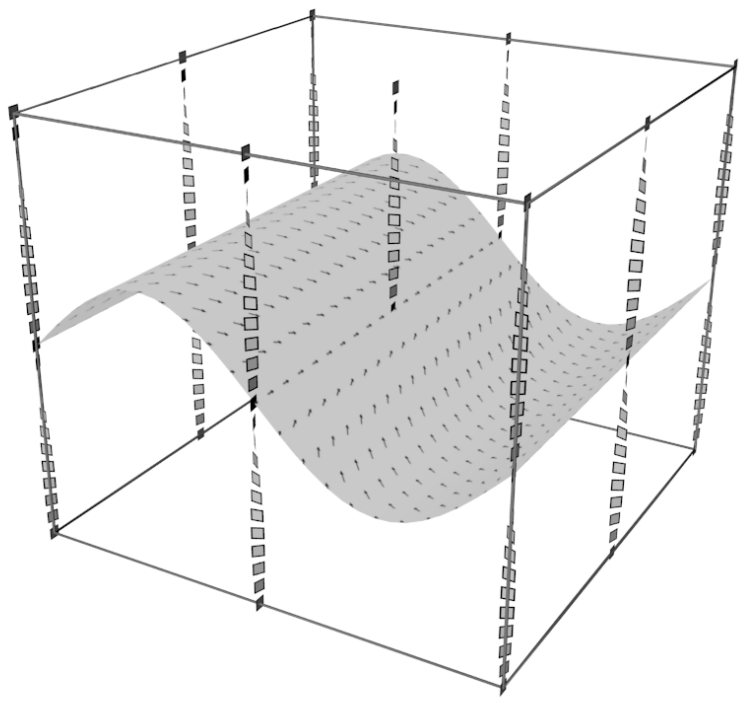

Figure 2.18: Perturbation of the non-convex torus of Figure 2.17 into a convex torus

closed orbit or a union of singularities and orbits connecting them.

The Poincaré-Bendixson theorem thus says that a singular foliation on a sphere satisfies the Poincaré-Bendixson property as soon as its singularities are isolated, see e.g. PdM82.

Proposition 26. Let $S$ be a surface in a contact manifold $(V, \xi)$. If the characteristic foliation $\xi S$ satisfies the Poincaré-Bendixson property then $S$ is $\xi$ convex if and only if $\xi S$ has neither degenerate closed leaves nor retrograde connections.

Genericity of $\xi$-convex surfaces then follows from Peixoto's theorem stating that Morse-Smale foliations are generic on surfaces, see PdM82 for a beautiful exposition of this result starting with the basic of dynamical systems. A foliation is Morse-Smale if

- it satisfies the Poincaré-Bendixson property,

- all its singularities are nodes or saddles,

- all its closed leaves are non-degenerate,

- it has no saddle connections.

Proof of Proposition 26. In the preceding sections, we have seen that the absence of degenerate closed leaves and retrograde connections is necessary for convexity. 
We now prove that it is sufficient when the Poincaré-Bendixson property holds. In this proof we assume that all singularities are nodes, saddles or saddlenodes. This is true for generic families of characteristic foliations with any number of parameters and is all we need in these lectures. In order to save some more words we will even pretend there are no saddle-nodes. The reader can replace any occurrence of the word "saddle" by "saddle or saddle-node" to get the more general proof.

During the discussion of obstructions to convexity, we have seen that singularities and closed leaves should be dispatched into $S_{+}$or $S_{-}$according to their signs. Another constraint comes from separatrices of saddles: since we want the characteristic foliation to go transversely out of $S_{+}$along $\Gamma$, stable separatrices of positive saddles and unstable separatrices of negative saddles cannot meet $\Gamma$.

So we build a subsurface $S_{+}^{\prime}$ of $S$ by putting a small disk around each positive singularity and narrow bands around positive closed leaves and stable separatrices of positive saddles. If all these elements are sufficiently small, the boundary of $S_{+}^{\prime}$ can be smoothed to a curve transverse to the characteristic foliation, see Figure 2.19. In addition one can find an area form on $S_{+}^{\prime}$ which is

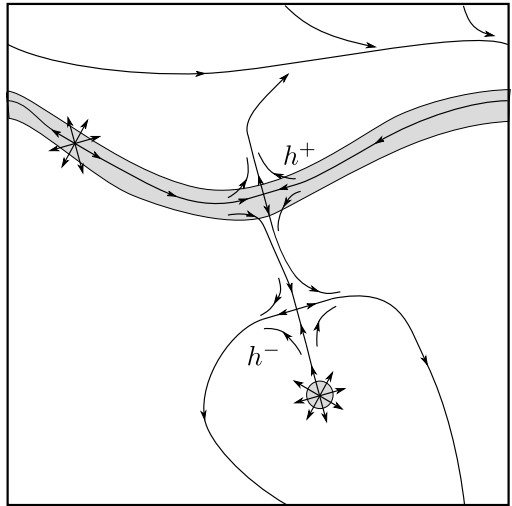

$S_{+}^{\prime}$

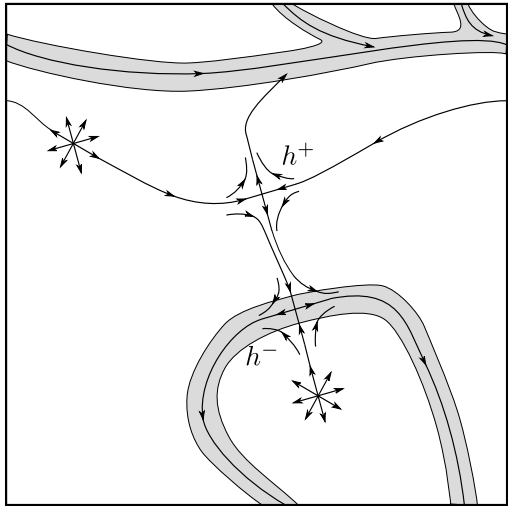

$S_{-}^{\prime}$

Figure 2.19: Construction of a dividing set on a torus. One can check that $\partial S_{+}^{\prime}$ and $\partial S_{-}^{\prime}$ are indeed isotopic among dividing curves.

expanded by $\xi S_{+}^{\prime}$. We can construct similarly a subsurface $S_{-}^{\prime}$ and a contracted area form on it. None of these subsurfaces is empty because of Stokes' theorem which guaranties that an area form on a closed surface is never exact.

Let $A$ be a component of the complement of $S_{+}^{\prime} \cup S_{-}^{\prime}$ in $S$. It has non-empty boundary and does not contain any singularity so $A$ is an annulus. In addition it does not contain any closed leaf so Poincaré-Bendixson's theorem guaranties that all leaves of the characteristic foliation entering $A$ along some boundary component leave it through the other boundary component. So we are indeed in the situation of Figure 2.7 and one can take the core of $A$ as a dividing curve. The corresponding subsurfaces $S_{ \pm}$then retract onto $S_{ \pm}^{\prime}$.

The proof above contains some useful information about how a dividing set can be recovered from the important features of the characteristic foliation so we record this in a definition and a corollary. 
Definition 27. Given a foliation $\mathscr{F}$ satisfying the Poincaré-Bendixon property, we denote by $G_{+}$(resp $G_{-}$) the union of repelling (resp attracting) closed leaves, of positive (resp negative) singularities and of the stable (resp unstable) separatrices of these singularities. The union $G_{+} \cup G_{-}$is called the Giroux graph of $\mathscr{F}$.

Note that the terminology graph is a little stretched since one can have separatrices accumulating on closed orbits (like in Figure 2.19) or on connected singularities so the Giroux graph equipped with the induced topology is not necessarily homeomorphic to a $C W$-complex of dimension one.

Corollary 28. If a characteristic foliation satisfies the convexity criterion of Proposition 26 and $G_{+} \cup G_{-}$is its Giroux graph then, for any dividing set, $S_{+}$ retracts on a regular neighborhood of $G_{+}$and $S_{-}$on a regular neighborhood of $G_{-}$.

\subsubsection{Giroux criterion and Eliashberg-Bennequin inequal- ities}

Until now, the discussion of this chapter does not make any distinction between tight and overtwisted contact structures. We now start to discuss how convex surfaces theory sees tightness.

Theorem 29 (Giroux criterion Gir01, Theorem 4.5a]). In a contact manifold $(V, \xi)$, a $\xi$-convex surface divided by some multi-curve $\Gamma$ has a tight neighborhood if and only if one of the following conditions is satisfied:

- no component of $\Gamma$ bounds a disk in $S$

- $S$ is a sphere and $\Gamma$ is connected.

The only application of this theorem we will present in detail is in the classification of tight contact structures on $\mathbb{S}^{3}$ (existence by Bennequin and uniqueness by Eliashberg). There we will only need that, if $S$ is a sphere, then it has a tight neighborhood only if its dividing set is connected. So we prove only this part of the theorem, we assume $S$ is a sphere and $\Gamma$ is not connected. Let $S^{\prime}$ be a component of $S \backslash \Gamma$ which is a disk and denote by $\gamma$ its boundary. Let $S^{\prime \prime}$ be the other component containing $\gamma$ in its boundary. Since $\Gamma$ is not connected, $S^{\prime \prime}$ has more boundary components. Using this, one can construct a foliation $\mathscr{F}$ on $S$ which is divided by $\Gamma$, has a circle of singularities $L$ in $S^{\prime \prime}$, is radial inside a disk bounded by $L$ and coincides with $\xi S$ outside $S^{\prime} \cup S^{\prime \prime}$, see Figure 2.20. In any neighborhood $U$ of $S$, the realization Lemma gives a surface $\delta_{1}(S)$ which has $\delta_{1}(\mathscr{F})$ as its characteristic foliation. Then $\delta_{1}(L)$ is the boundary of an overtwisted disk contained in $\delta_{1}(S)$ hence in $U$.

An important direct application of the Giroux criterion is Giroux's proof of the following constraint on the Euler class of a tight contact structure (originally due to Eliashberg). We will not use it in those notes but include it here since it now comes for free.

Theorem 30 (Eliashberg-Bennequin inequality Eli92]). Let $(M, \xi)$ be a $3-$ dimensional contact manifold. If $\xi$ is tight and $S$ is a closed surface embedded in $M$ then the Euler class of $\xi$ satisfies the following inequality:

$$
|\langle e(\xi), S\rangle| \leq \max (0,-\chi(S))
$$



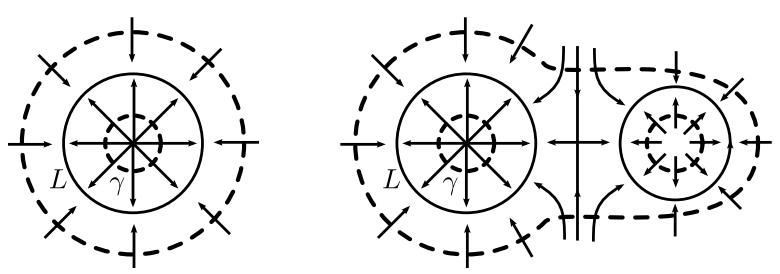

Figure 2.20: Characteristic foliations for the Giroux criterion. The dividing set $\Gamma$ is dashed. On the left-hand side one has the simplest case when $S^{\prime \prime}$ is an annulus. On the right hand-side one sees a possible foliation when $S^{\prime \prime}$ has one more boundary component (on the right). Note that the disk bounded by the small component of $\Gamma$ on the right may contain more components of $\Gamma$. The extension to more boundary components uses the same idea.

Proof. Using genericity of $\xi$-convex surfaces, one can homotop $S$ until it is $\xi-$ convex. This does not change the Euler class which can now be evaluated as $\chi\left(S_{+}\right)-\chi\left(S_{-}\right)$since singularities are distributed among $S_{+}$and $S_{-}$according to their signs. If $S$ is a sphere then the Giroux criterion says that both $S_{+}$and $S_{-}$are disks so $\langle e(\xi), S\rangle=0$ and the inequality is proved. So suppose now that $S$ has positive genus. The Giroux criterion says that no connected component of $S_{+}$or $S_{-}$is a disk. This implies that both $\chi\left(S_{+}\right)$and $\chi\left(S_{-}\right)$are negative. Hence both $\chi\left(S_{+}\right)-\chi\left(S_{-}\right)$and $-\chi\left(S_{+}\right)+\chi\left(S_{-}\right)$are less than $-\chi\left(S_{+}\right)-\chi\left(S_{-}\right)$ which is $-\chi(S)$. 


\section{Chapter 3}

\section{Bifurcations and first classification results}

The goal of this chapter is to prove that any tight contact structure on $\mathbb{S}^{3}$ has to be isotopic to the standard contact structure and that the later is indeed tight. We will not give the original proofs due to Eliashberg Eli92 and Bennequin Ben83 respectively. We will rather use the technology of $\xi$-convex surfaces to prove them. These proofs were obtained by Giroux along its way towards more general classification results in Gir00. The classification result is a comparatively easy special case of Giroux's preparation Lemma [Gir00, Lemma 2.17] while the tightness result follows from the bifurcation lemmas Gir00, Lemmas 2.12 and 2.14].

\subsection{The elimination lemma}

In the characteristic foliation of a surface, a saddle and a node are said to be in elimination position if they have the same sign and there is a leaf from one to the other. Such a leaf is called an elimination arc. Giroux's elimination lemma in its simplest form says one can perturb the surface to replace a neighborhood of the elimination arc by a region without singularity as in Figure 3.1

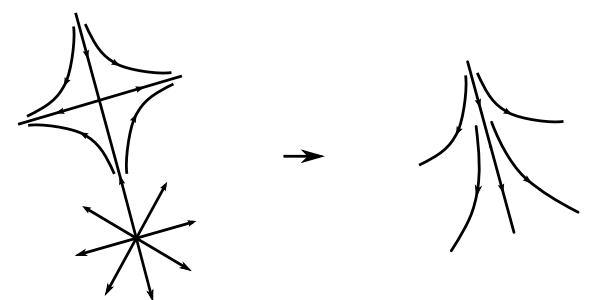

Figure 3.1: Elimination of a pair of singular points.

For the classification of tight contact structures on $\mathbb{S}^{3}$ we will need a version of this process which keeps neighboring surfaces under control. 


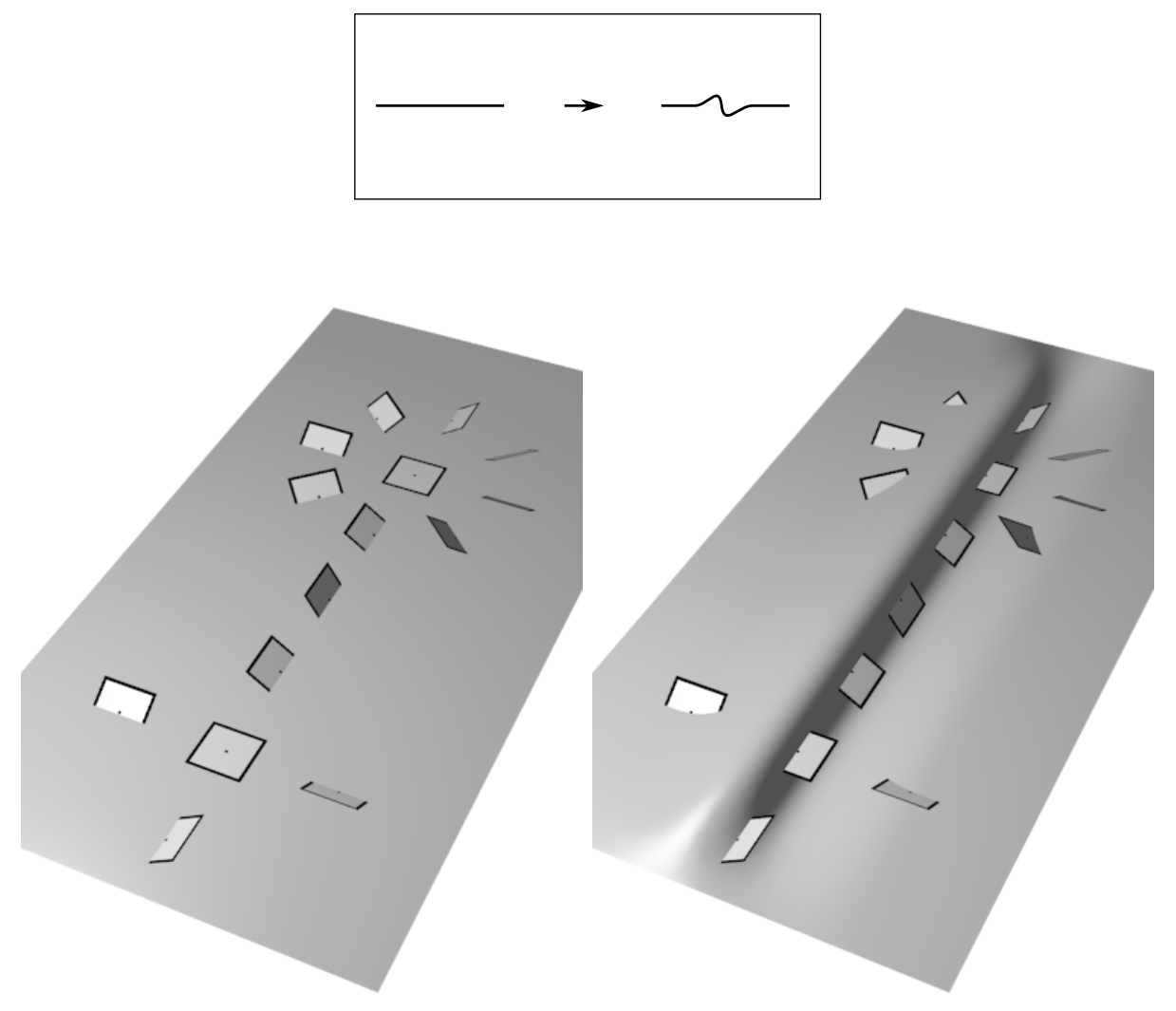

Figure 3.2: The elimination move. The top box shows the move transverse to the elimination arc seen as the middle point of the segment. This move is cut off in the longitudinal direction. 
We do not need much control though and the following version is simpler than Gir00, Lemma 2.15] which is needed for the classification of tight contact structures on torus bundles.

Let $\xi$ be a contact structure on $S \times[-1,1]$ and set $S_{t}:=S \times\{t\}$. Suppose a node $e_{0}$ and a saddle $h_{0}$ are in elimination position on $S_{0}$. This configuration is stable so it persists for $t$ in some interval $(-\varepsilon, \varepsilon)$. Let $C_{t}$ denote a continuous family of elimination arcs between $e_{t}$ and $h_{t}$ on $S_{t}$.

Lemma 31 (Giroux elimination lemma). Let $\delta$ be a positive number smaller than $\varepsilon$. Let $U$ a neighborhood of $\bigcup_{|t|<\delta} C_{t}$ intersecting each $S_{t}$ in a disk $D_{t}$ whose characteristic foliation is as in the left hand side of Figure 3.1. One can deform $\xi$ in $U$ such that $\xi D_{t}$ has:

- no singular point when $|t|<\delta$,

- a saddle-node when $|t|=\delta$,

- a pair of singularities in elimination position when $|t| \in(\delta, \varepsilon)$.

In addition, one can impose that separatrices facing the elimination arc are connected to the same points of $\partial D_{t}$ as before the deformation, see Figure 3.3.

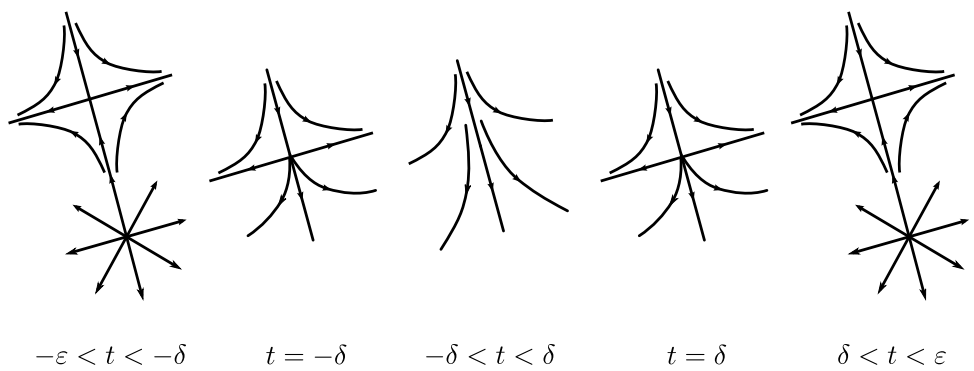

Figure 3.3: Elimination in a family.

The corresponding manipulation transverse to the elimination arc is explained in Figure 3.4

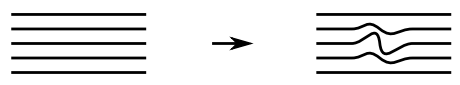

Figure 3.4: The elimination move in family. The left hand-side shows the original surfaces $S_{t}$ stacked. The right hand-side performs the elimination, compare with top of Figure 3.2

\subsection{Thickened spheres and Eliashberg uniqueness}

The goal of this section is to explain Giroux's proof of the classification of tight contact structures on $\mathbb{S}^{3}$. 
Theorem 32 (Eliashberg Eli92). Any tight contact structure on $\mathbb{S}^{3}$ is isotopic to the standard one.

By definition of contact structures, one can assume that $\mathbb{S}^{3}$ is the union of two standard balls and a thickened sphere with standard $\xi$-convex boundary as in Figure 2.2. This allows in particular to apply the following proposition.

Proposition 33. Let $\xi$ be a tight contact structure on a thickened sphere $S \times$ $[0,1]$. If $S_{0}$ and $S_{1}$ are $\xi$-convex then $\xi$ is isotopic relative to the boundary to a contact structure $\xi^{\prime}$ such that all spheres $S_{t}$ are $\xi^{\prime}$-convex.

Proof. First note that tightness prevents the apparition of any closed leaf in any $\xi S_{t}$ since it would bound an overtwisted disk. Then we need some theory of one-parameter families of singular foliations on the sphere Sot74. Specifically, one can assume that each $\xi S_{t}$ has finitely many singularities and at worse a saddle connection or a saddle-node (but not both at the same time). Note that finiteness of saddle connections can be achieved by perturbation thanks to the absence of closed leaves (compare Figure 3.10). Using this, the PoincaréBendixson theorem and the criterion of Proposition 26, one can see that all surfaces $S_{t}$ are $\xi$-convex except for finitely many $t_{1}, \ldots, t_{k}$ where:

- all singularities of $\xi S_{t_{i}}$ are saddles or nodes

- there is exactly one saddle connection on $\xi S_{t_{i}}$ and it is retrograde,

see Figure 3.5 for an example.

We will now modify $\xi$ near each $S_{t_{i}}$ in order to make all $S_{t} \xi$-convex. Since we know closed leaf or non-trivial recurrence cannot arise, it suffices to get rid of retrograde saddle connections. We concentrate on one $t_{i}$ at a time. Let $\varepsilon$ be a small positive number such that $\xi S_{t}$ does not change up to homeomorphism when $t$ is either in $\left[t_{i}-\varepsilon, t_{i}\right)$ or $\left(t_{i}, t_{i}+\varepsilon\right]$. In particular the positive part $G^{+}$of the Giroux graph deforms by isotopy in each of these intervals. Theorem 29, the Giroux criterion, and the link between the Giroux graph and the dividing set explained in Corollary 28 guarantee that $G^{+}$is a tree in each interval. It implies that we can find elimination arcs between all positive saddles and all but one positive nodes without using the separatrix which enters the saddle connection at $t_{i}$ (recall in particular that the number of vertices in a tree is exactly the number of edges plus one).

We now use Lemma 31, the elimination lemma, to get rid of all positive saddles for $t$ in $[t-\delta, t+\delta]$ for some positive $\delta$ smaller than $\varepsilon$, see Figure 3.6 .

Before continuing the proof of the theorem, we note two properties of the sphere which were somehow surreptitiously used in the above proof. After the elimination of the retrograde connections we needed the fact that no closed leaves could appear, this is due to Schönflies theorem which would have provided an overtwisted disk. We also needed the Poincaré-Bendixson theorem to prevent the apparition of non-trivial recurrence. Suppose one tries to use the elimination lemma to get rid of the bifurcation of Figure 3.9 (which is bound to fail since the isotopy class of the dividing set changes during this bifurcation). If one gets rid of both saddles then degenerate leaves arise. If one gets rid of one saddle only (like we did for the sphere) then non-trivial recurrence appear: we get a Cherry flow on the torus, see $\mathrm{PdM} 82$. 

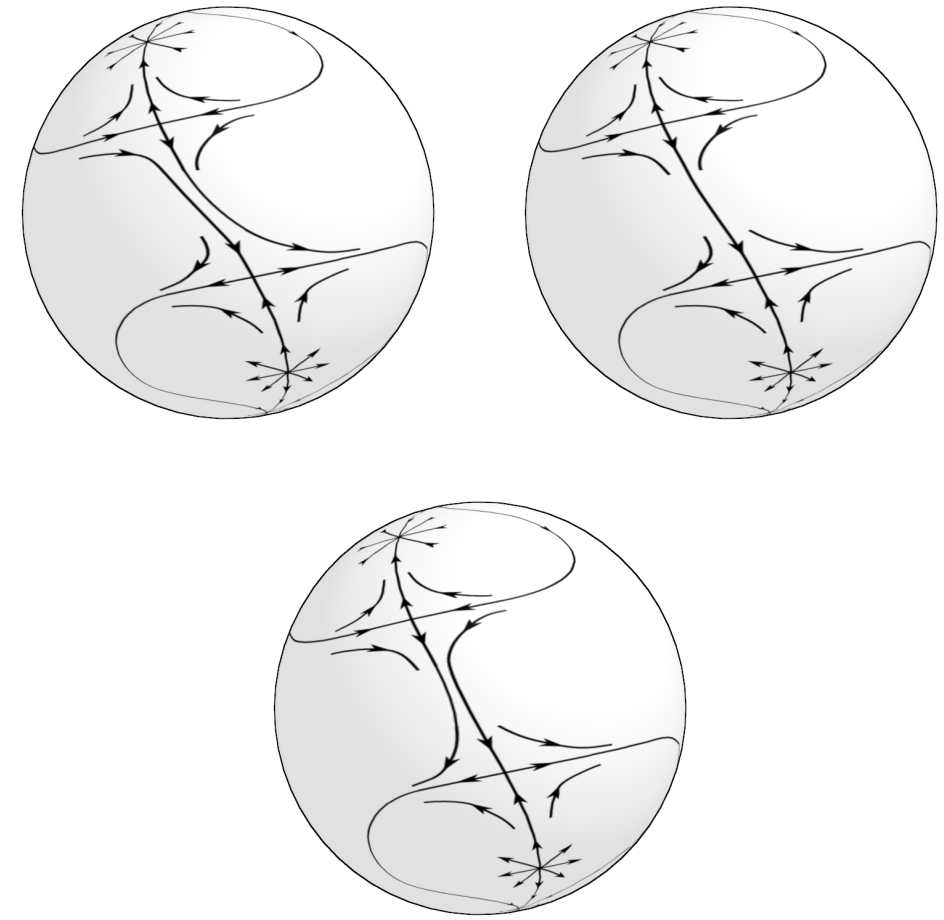

Figure 3.5: Original movie 

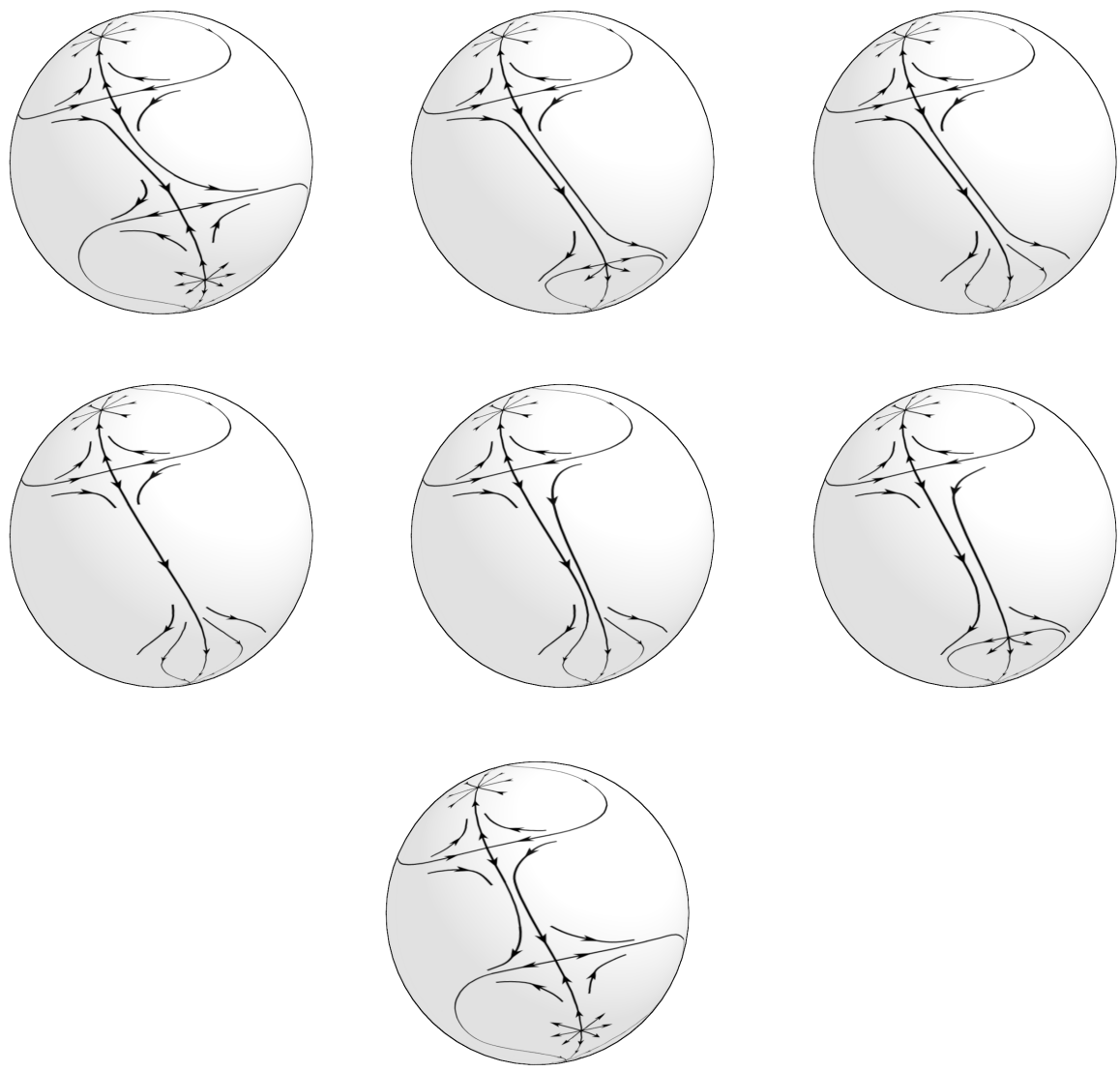

Figure 3.6: Movie after elimination. The first picture is the same as in Figure 3.5 then a pair of singularity is replaced by a saddle-node then it disappears. The fourth picture corresponds to the central picture of Figure 3.5 but there is no more positive saddle so no saddle connection. The eliminated pair returns in the sixth picture as a saddle-node and the final picture is the same as in Figure 3.5 
The proof of Theorem 32 now follows from Giroux's uniqueness lemma which allows to replace the contact structure obtained on the thickened sphere of the previous proposition by the model.

Lemma 34 (Uniqueness lemma Gir00, Lemma 2.7]). Let $\xi_{0}$ and $\xi_{1}$ be two contact structures printing the same characteristic foliations on the boundary of $S \times[0,1]$. If there is a continuous family of multi-curves $\Gamma_{t}$ dividing both $\xi_{0} S_{t}$ and $\xi_{1} S_{t}$ then $\xi_{0}$ and $\xi_{1}$ are isotopic relative to the boundary.

The proof of this lemma is similar to the ones of the previous chapter but the path of contact structures is less obvious.

We now explain how to get the classification of tight contact structures on $\mathbb{S}^{2} \times \mathbb{S}^{1}$ without extra effort. Let $\xi$ be one of them and fix some $S=\mathbb{S}^{2} \times\left\{\theta_{0}\right\}$. Using genericity of $\xi$-convex surfaces, we can perturb $\xi$ to make $S$ convex. Then the Giroux criterion tells us that its dividing set is connected. Using the realisation lemma, we change $\xi$ by isotopy until $\xi S$ is standard, ie as in Figure 2.2 We can then remove a homogeneous neighborhood of $S$ and we are back to a thickened sphere where we can apply Proposition 33 and the uniqueness lemma.

\subsection{Bifurcation lemmas}

We now consider a general closed surface $S$ and any contact structure $\xi$ on $S \times I$ for some interval $I$. For each $t$ in $I$, one has the surface $S_{t}:=S \times\{t\}$ and its characteristic foliation $\xi S_{t}$. If some $S_{t_{0}}$ is not $\xi$-convex then the characteristic foliations for $t$ close to $t_{0}$ are not all $C^{1}$-conjugate to $\xi S_{t_{0}}$, otherwise the global reconstruction lemma (Lemma 12 would give a contradiction. We will now try to understand what really happens when this lack of $\xi$-convexity is explained by the obstructions we discussed in the previous chapter, ie it comes from a degenerate closed leaf or a retrograde connection. We will see in particular that the bifurcation is much sharper than expected: no foliation $\xi S_{t}$ is even $C^{0}$ conjuguate to $\xi S_{t_{0}}$ for $t$ in a punctured neighborhood of $t_{0}$. Better, we will get a very precise description of what happens.

\section{The birth/death lemma}

Let $L$ be a degenerate closed leaf of the characteristic foliation $\xi S_{t}$. This means that the Poincaré return map on any curve transverse to $L$ is tangent to the identity. One says that $L$ is positive (resp negative) if the second derivative of this map is positive (resp negative) at the intersection point between $L$ and the transverse curve. If $L$ is either positive or negative then one says that it is weakly degenerate.

Lemma 35 (Birth/Death Lemma Gir00, Lemma 2.12]). A positive (resp negative) degenerate closed orbit indicates the birth (resp death) of a pair of nondegenerate closed leaves when $t$ increases.

See Figure 3.7 for examples of these situations on a thickened torus $T \times[0,1]$. Looking at these pictures it is easy to prove a weak form of the birth-death lemma which already shows how the contact condition enters. Since the contact structure is transverse to all tori $T_{t}, t \in[0,1]$, one can lift $\partial_{t}$ to a vector field 

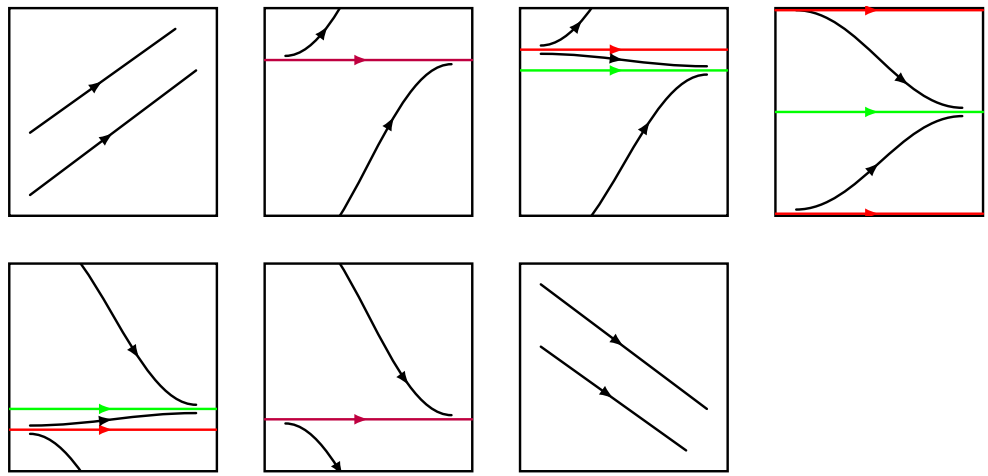

Figure 3.7: Birth and death of closed leaves on a torus.

tangent to $\xi$. The flow of this lift defines a new product structure on $T \times[0,1]$ without changing the movie of singular foliations $\xi T_{t}$ up to diffeomorphism. So one can assume that all intervals $I_{p}=\{p\} \times[0,1]$ are Legendrian. If we think of foliations $\xi T_{t}$ as living all on $T$ then the contact condition is equivalent to asking that, at each point $p, \xi T_{t}(p)$ rotates clockwise as $t$ increases. Indeed, if $x$ and $y$ are coordinates on $T$, there is a function $\theta$ such that

$$
\xi=\operatorname{ker}(\cos \theta(x, y, t) d x-\sin \theta(x, y, t) d y) .
$$

The contact condition is then equivalent to $\partial_{t} \theta>0$, compare with the proof of the Darboux-Pfaff theorem (Theorem 2).

Now the second picture in Figure 3.7 shows a positive degenerate orbit $L$ in some $\xi T_{t_{0}}$. Let $A$ be a small annulus around $L$. Along $L$, the slope of $\xi T_{t_{0}}$ is zero and it is positive in $A \backslash L$. So, for $t<t_{0}$ it was everywhere positive in $A$ and there were no closed leaf at all in $A$. For $t>t_{0}$, the slope becomes negative along $L$ and stays positive along the boundary of $A$. Then the complement of $L$ in $A$ is made of two (half-open) annuli whose boundary are transverse to $\xi T$, see Figure 3.8. The Poincaré-Bendixson theorem guaranties that each of these two sub-annuli contain at least one closed leaf for $t>t_{0}$ sufficiently close to $t_{0}$.

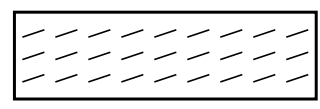

$t_{0}-\varepsilon$

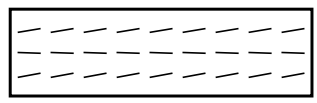

$t_{0}$

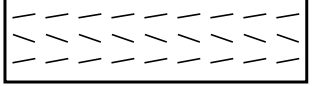

$t_{0}+\varepsilon$

Figure 3.8: Birth of at least a pair of periodic orbits. The annulus $A$ is obtained by gluing left and right. The circle $L$ is at mid-height of each annulus.

So we proved the following weak version of the birth/death lemma which will be sufficient for our purposes: if there is a positive degenerate closed orbit $L$ at time $t_{0}$ then there is an annulus $A$ around $L$ and some positive $\varepsilon$ such that there is no closed leaves in $A$ for $t$ in $\left(t_{0}-\varepsilon, t_{0}\right)$ and at least two for $t$ in $\left(t_{0}, t_{0}+\varepsilon\right)$. The death case on the bottom row of Figure 3.7 is explained similarly. Note that nothing required $T$ to be a torus in this explanation, one only has to work near $L$. 


\section{The crossing lemma}

Lemma 36 (Crossing Lemma Gir00, Lemma 2.14]). Assume that there is a retrograde connection at time $t_{0}$. For $t$ close to $t_{0}$, there is a negative singularity $b_{t}^{-}$, a positive one $b_{t}^{+}$, an unstable separatrix $c_{t}^{-}$of $b_{t}^{-}$and a stable one $c_{t}^{+}$of $b_{t}^{+}$such that $c_{t_{0}}^{-}=c_{t_{0}}^{+}$.

For $t$ close to $t_{0}$, one can track separatrices using their intersection with an oriented curve positively transverse to $\xi S_{t}$. Then, for $t<t_{0}$ (resp $\left.t>t_{0}\right)$, the separatrix $c_{t}^{-}$is below (resp above) $c_{t}^{+}$.

Figure 3.9 shows a retrograde saddle connection on a torus obtained by gluing top/bottom and left/right. Singularities in the lower part are negative while those in the upper part are positive. The saddle connection is marked by an arrow. The crossing Lemma tells us that the negative separatrix has to turn to its right after the connection.
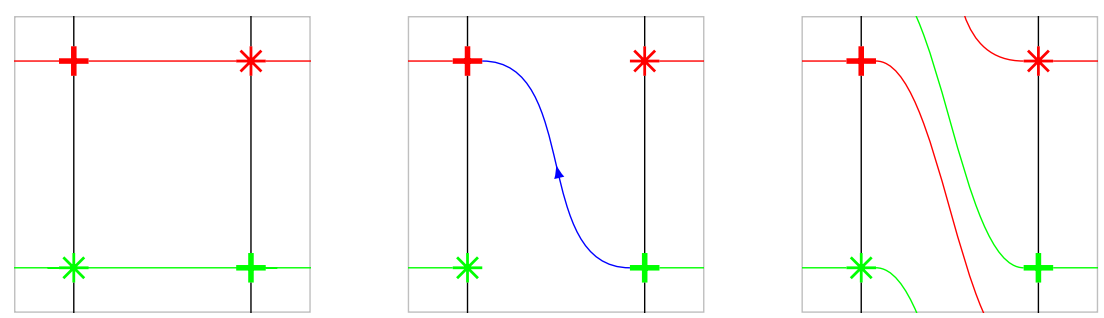

Figure 3.9: Retrograde saddle connection on a torus.

The proof of the crossing lemma is rather delicate so we will only try to go as far as explaining how the contact condition and the fact that the connection is retrograde can enter the discussion. Each time we drop the $t$ subscript it means $t=t_{0}$. Also we set $c=c^{+}=c^{-}$. Compared to the situation of the birth/death lemma, there is no hope to have a neighborhood $S \times[0,1]$ with $[0,1]$ tangent to $\xi$ near $c$ since $\xi$ is tangent to $S$ at $b^{ \pm}$. However we will find at least one point on $c$ where the characteristic foliation has to turn clockwise. If $Y_{t}$ is a vector field defining $\xi S_{t}$, the contact condition $(\star)$ can be expressed as: $u_{t} \operatorname{div} Y_{t}-d u_{t}\left(Y_{t}\right)+\dot{\beta}_{t}\left(Y_{t}\right)>0$. The sign of singularities is the sign of $u_{t}$ so $u\left(b^{-}\right)<0$ and $u\left(b^{+}\right)>0$. Hence there is some point $p$ on $c$ such that $u(p)=0$ and $d u(Y) \geq 0$. Here we used that $c$, hence $Y$, is oriented from $b^{-}$to $b^{+}$. At $p$, the contact condition becomes $\dot{\beta}(Y)>d u(Y)$ so $\dot{\beta}(Y)>0$. This is the announced rotation. Since $\beta(Y)=0$, we have that, at $p, \xi S_{t}$ is positively transverse to $c$ for $t>t_{0}$ and negatively transverse for $t<t_{0}$. Of course this observation is very far from proving the crossing lemma, see Gir00, Lemma $2.14]$ for the full story.

\subsection{Bennequin's theorem}

The goal of this section is to prove that the standard contact structure on $\mathbb{R}^{3}$ is tight. This was originally proved by Bennequin, without the word tight which was introduced by Eliashberg.

Suppose there is an overtwisted disk in the standard contact structure on $\mathbb{R}^{3}$. Since it is compact, it is contained in some finite radius ball. We can also 
assume it misses a small ball around the origin (for instance we can use the contact vector field $\partial_{z}$ to push it upward until this is true). Recall we saw in Example 16 there is a contact vector field $X$ on $\mathbb{R}^{3}$ which is transverse to all Euclidean spheres around the origin. So these spheres are all $\xi$-convex and divided by the equator $\{z=0\}$ where $X$ is tangent to $\xi$. The above discussion shows that Bennequin's theorem is a consequence of the following statement.

Theorem 37 (Bennequin seen by Giroux Gir00, Theorem 2.19]). Let $\xi$ be a contact structure on a thickened sphere $S \times[-1,1]$. If all spheres $S_{t}$ are $\xi$-convex with connected dividing set then $\xi$ is tight.

\section{Families of movies}

In order to prove Theorem 37, we first need some preparations from dynamical systems. Suppose that $\xi_{0}$ and $\xi_{1}$ are two contact structures which print generic movies on $S \times[-1,1]$. If they are isotopic, one gets a 2-parameters family $\xi_{s} S_{t}$ of characteristic foliations. Thom transversality and a little bit of normal form theory tells us that we can perturb the family until all these foliations have finitely many singularities which are either nodes, saddles or saddle-nodes. Further perturbations allow to make sure that all closed leaves have a Poincaré return map which is at worse tangent to the identity up to order 2 , the worse case happening only for isolated values of $(s, t)$.

Up to this point there was nothing specific to the sphere. The first special property of $\mathbb{S}^{2}$ which is crucial in the following is the Poincaré-Bendixson theorem which says that, since we have isolated singularities for all our foliations, the Poincaré-Bendixson property automatically holds. In particular we can apply the convexity criterion of Proposition 26 . In the square $[0,1] \times[-1,1]$ the set $\Omega$ of points $(s, t)$ such that $S_{t}$ is $\xi_{s}$-convex is a dense open set. We denote by $\Sigma$ the complement of $\Omega$. It is a union of injectively immersed submanifolds of $[0,1] \times[-1,1]$. In codimension 1 , one sees:

- $\Sigma_{\mathrm{dl}}^{1}$ where the characteristic foliation has a single weakly degenerate closed leaf and no retrograde saddle connection and no degenerate singularity, see Figure 2.12

- $\Sigma_{\mathrm{sc}}^{1}$ where the characteristic foliation has a single retrograde saddle connection and no degenerate closed leaf or singularity, see Figure 3.9

The bifurcation lemmas imply that these two subsets are injectively immersed submanifold of the square transverse to the $t$ direction. In addition, the bifurcation lemmas imply that components of $\Sigma_{\mathrm{dl}}^{1}$ can accumulate only on $\Sigma_{\mathrm{sc}}^{1}$, see Figure 3.10 for an example of accumulation. We set $\Sigma^{1}=\Sigma_{\mathrm{dl}}^{1} \cup \Sigma_{\mathrm{sc}}^{1}$.

The accumulation of retrograde saddle connections in Figure 3.10 is not a phenomena which we can get rid of by perturbation: it is structurally stable in a 1-dimensional family, see [Sot74]. However, Giroux's discretization lemma Gir01b, lemma 15] states that any contact structure on the product $F \times I$ of a closed surface and an interval with convex boundary is isotopic relative to the boundary to a contact structure such that only finitely many $F_{t}$ are non-convex. This isotopy cannot be made arbitrarily small. It uses first the dynamics banalization lemma Gir00, Lemma 2.10] which gets rid of nontrivial recurrence and then replaces degenerate leaves with retrograde saddle connexions. Both moves are non-perturbative.

In codimension 2, one sees: 

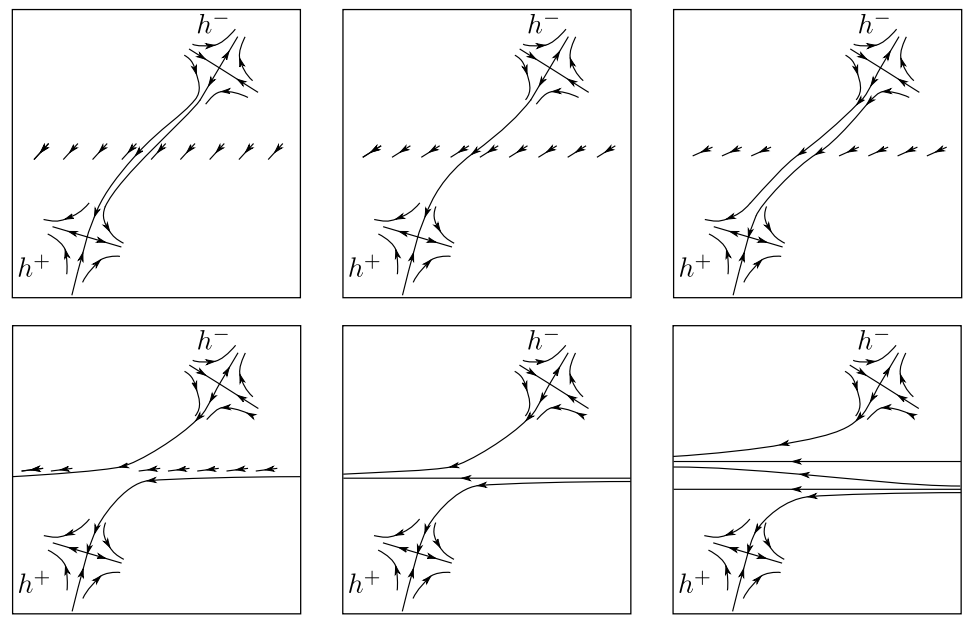

Figure 3.10: Saddle connections accumulating a degenerate closed leaf. This is a movie of characteristic foliations on an annulus obtained by gluing the left and right sides of each square. A degenerate closed leaf is appearing in the middle. Leaves spiral more and more in this region, resulting in infinitely many retrograde saddle connections.

- $\Sigma^{11}$ where two codimension one strata intersect transversely, see Figure 3.11 and also Figure 2.16 for a realistic view of the central picture in the case of example 23.

- $\Sigma_{\mathrm{sc}}^{2}$ where there is a retrograde connection between a saddle and a saddlenode. These points adhere to exactly one stratum in $\Sigma_{\mathrm{sc}}^{1}$, this typically happens in the proof of the classification on $S^{3}$ as an intermediate step between Figures 3.5 and 3.6

- $\Sigma_{\mathrm{dl}}^{2}$ where there is a degenerate orbit corresponding to the fusion of two components of $\Sigma_{\mathrm{dl}}^{1}$, see Figure 3.12 for the picture in the $(s, t)$ square and Figure 3.13 for the corresponding foliations.

\section{Proof core}

We now prove Theorem 37. Suppose there is some overtwisted disk in $(S \times$ $[-1,1], \xi)$. Then there is some isotopy relative to the boundary bringing this disk onto the middle sphere $S_{0}$. So this isotopy sends $\xi_{0}=\xi$ to a contact structure $\xi_{1}$ such that $S_{0}$ contains an overtwisted disk. Then it can be modified in the same way genericity of convex surfaces is proved until $S_{0}$ is $\xi_{1}$-convex and divided by a disconnected curve (use Corollary 28 to understand dividing sets here). We can perturb $\xi_{1}$ to make sure it also prints a generic movie of characteristic foliations and perturb the isotopy to be in the situation of the preceding discussion on families of movies.

The set $\Omega$ of $(s, t)$ such that $S_{t}$ is $\xi_{s}$-convex is the disjoint union of $\Omega_{c}$ corresponding to connected dividing sets and $\Omega_{d}$ corresponding to disconnected ones. 

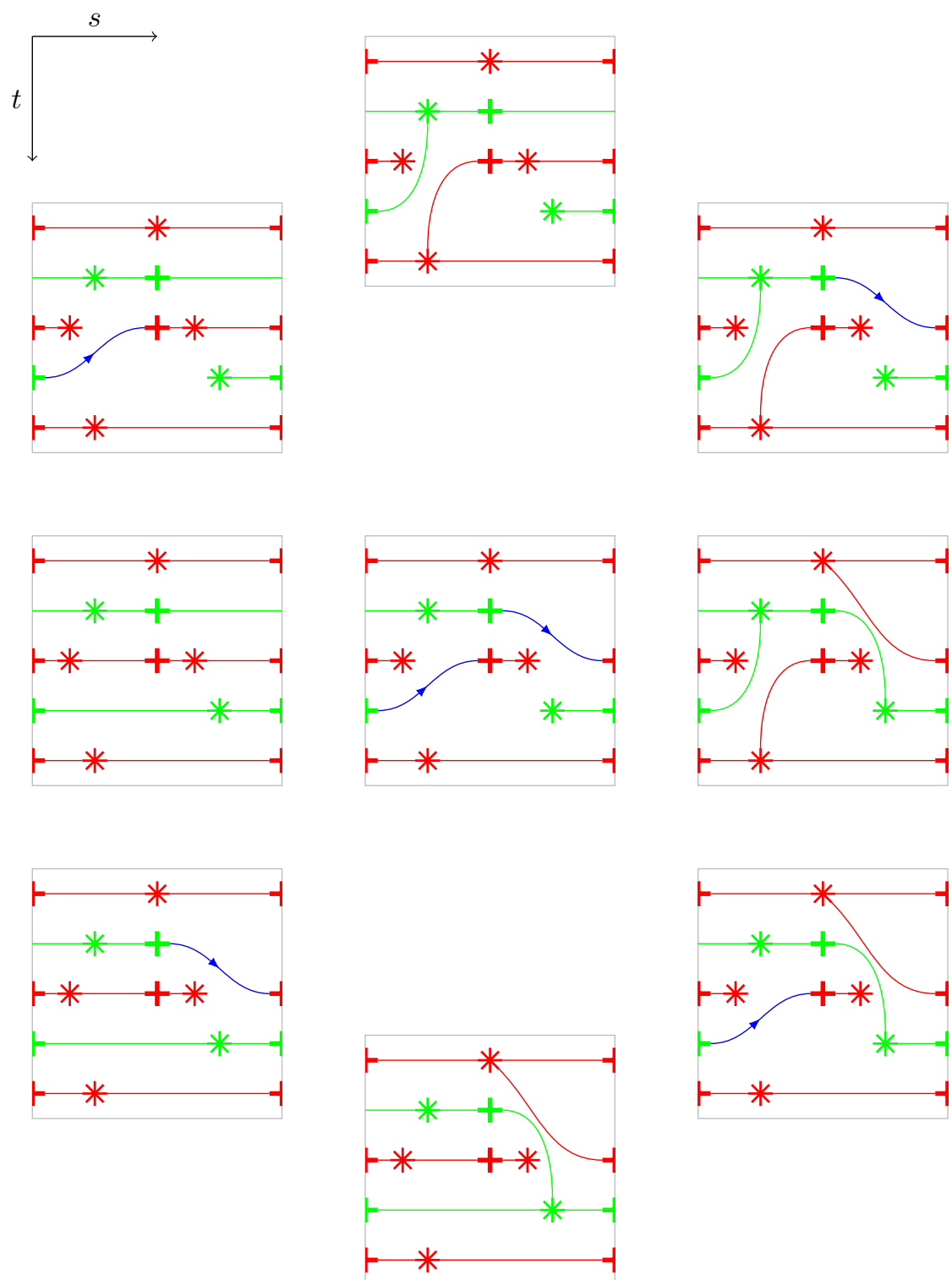

Figure 3.11: Intersection of two strata of retrograde saddle connections on a torus. It is a good exercise to draw the Giroux graphs of all convex surfaces appearing to see the non-trivial effect of this codimension 2 phenomenon on the dividing sets, contrasting with the discussion below. 


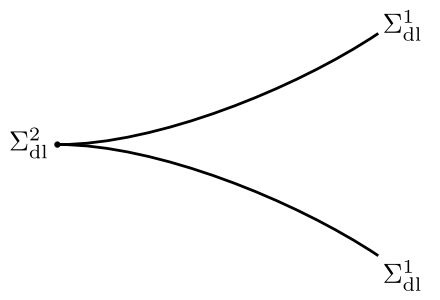

Figure 3.12: The central point is in $\Sigma_{\mathrm{dl}}^{2}$. It corresponds to a degenerate closed leaf with $\pi^{\prime \prime}(0)=0$ but $\pi^{(3)}(0)<0$, see Figure 3.13 for the corresponding foliations.

In addition, we know by construction that $\Omega_{d}$ intersects the right vertical edge $\{s=1\}$ so it is not empty. But it does not intersect the left edge $\{s=0\}$ by hypothesis of the theorem. More precisely, we can assume the closure of $\Omega_{d}$ does not meet $\{s=0\}$ so the minimum $s_{0}$ of its projection to $[0,1]$ is positive. Choose $t_{0}$ such that $\left(s_{0}, t_{0}\right)$ is in the closure of $\Omega_{d}$.

The point $\left(s_{0}, t_{0}\right)$ cannot be in:

- $\Sigma^{1}$ because the later is transverse to the $t$ direction so components of $\Omega$ adjacent to a point $(s, t)$ in $\Sigma^{1}$ project to neighborhoods of $s$

- $\Sigma_{\mathrm{sc}}^{2}$ because each point $(s, t)$ in $\Sigma_{\mathrm{sc}}^{2}$ adheres to only one component of $\Sigma_{\mathrm{sc}}^{1}$ so the intersection between $\Omega$ and a small disc around $(s, t)$ is connected and projects to a neighborhood of $s$.

- $\Sigma_{\mathrm{dl}}^{2}$ because all components of $\Omega$ touching $\Sigma_{\mathrm{dl}}^{2}$ are in $\Omega_{d}$ because the corresponding foliations have closed leaves.

- any point $\Sigma^{11}$ involving degenerate closed leaves, again because strata in $\Sigma_{\mathrm{dl}}^{1}$ are transverse to the $t$-direction and indicate birth or death of stable closed leaves giving disconnected dividing sets.

The only configuration which really needs to be carefully ruled out is that of points in $\Sigma^{11}$ involving only $\Sigma_{\mathrm{sc}}^{1}$ like in figure 3.14 In this situation $\xi_{s_{0}} S_{t_{0}}$ has two retrograde saddle connections which happen on different surfaces $S_{t}$ for $s$ in a punctured neighborhood of $s_{0}$ and get swapped when $s$ goes through $s_{0}$, as in Figure 3.11. Note that characteristic foliations around $\left(s_{0}, t_{0}\right)$ have no closed leaf and we can also assume they do not have other saddle connections that the ones we explicitly study.

To $\xi_{s} S_{t}$ we associate the oriented graph $\Gamma_{+}(s, t)\left(\operatorname{resp} . \Gamma^{-}(s, t)\right)$ whose vertices are positive nodes and edges are the stable separatrices of positive saddles (resp. negative saddles). Since we do not have any closed leaf or degenerate singularities near $\left(s_{0}, t_{0}\right), \Gamma_{+}$coincides as a set with $G_{+}$from definition 27 and $\Gamma^{-}$is somehow dual to $G_{-}$. So, according to Corollary 28, when $S_{t}$ is $\xi_{s}$-convex, there is a regular neighborhood of $\Gamma_{+}(s, t)$ whose boundary divides $\xi_{s} S_{t}$. Because $S$ is a sphere, we then get that $(s, t)$ is in $\Omega_{c}$ if and only if $\Gamma_{+}(s, t)$ is a tree (ie a closed connected and simply connected graph). We want to use the crossing lemma to understand how the graph changes when a retrograde saddle connection happens, see Figure 3.15 . 

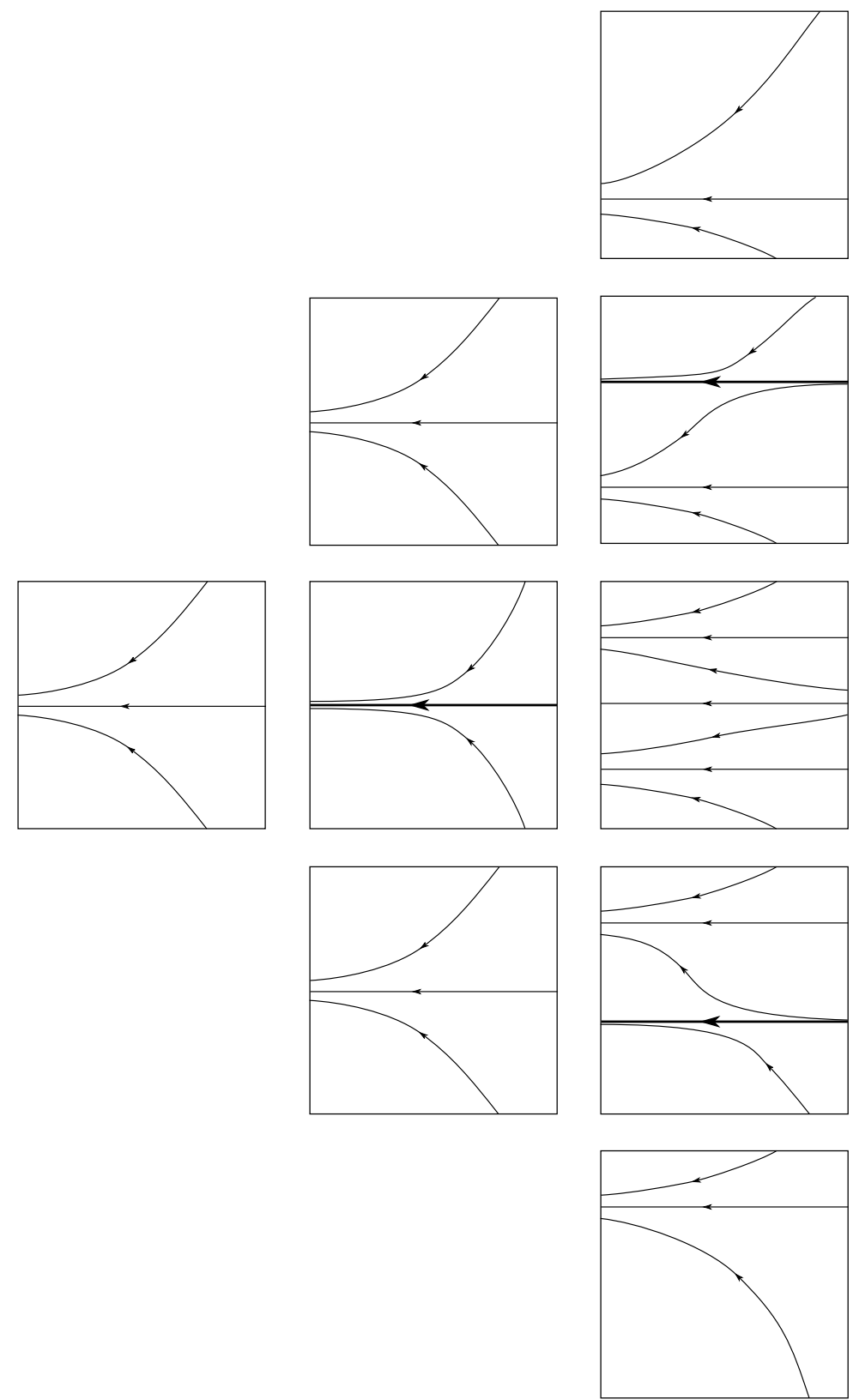

Figure 3.13: Foliations corresponding to the strata of Figure 3.12 Left and right of each square are glued to get an annulus. Thick closed leaves are the degenerate ones. The central picture corresponds to the annihilation of a birth and a death of non-degenerate closed leaves. 


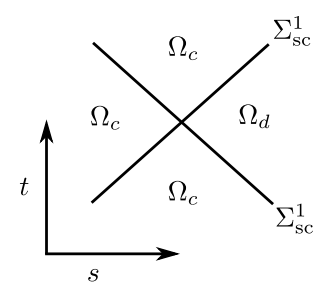

Figure 3.14: The situation we must rule out for Bennequin's theorem
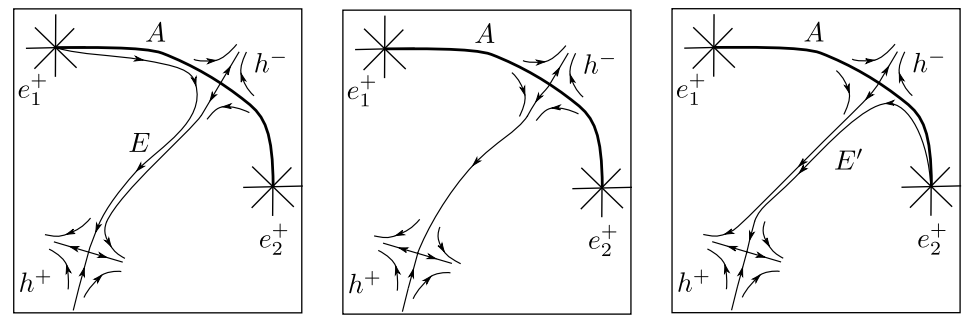

Figure 3.15: Anatomy of a retrograde saddle connection

First we remark that, if we focus on a sufficiently small neighborhood of $\left(s_{0}, t_{0}\right)$ in parameter space, the graph $\Gamma^{-}(s, t)$ deforms by isotopy so we can assume it does not depend on $s$ and $t$. The same is true for $\Gamma_{+}(s, t)$ as long as we stay in the complement of $\Sigma$. Suppose now there is a saddle connection involving a negative saddle $h^{-}$. Let $A$ be the closure of the union of its stable separatrices. The unstable separatrix of $h^{-}$entering the saddle connection coorients $A$ and, together with the orientation of $S$, this orients $A$. We denote by $o(A)$ and $d(A)$ the origin and destination of $A$.

During a bifurcation, exactly one edge $E$ of $\Gamma_{+}$changes. After the bifurcation, the edge $E$ is replaced by an edge $A(E)$ which is obtained from the concatenation of $E$ and $A$ by a small push towards the right which makes it avoid $o(A)$, see Figure 3.16 which also explains how these things will be drawn schematically in the following. Note that the edge $E$ is the edge which is imme-

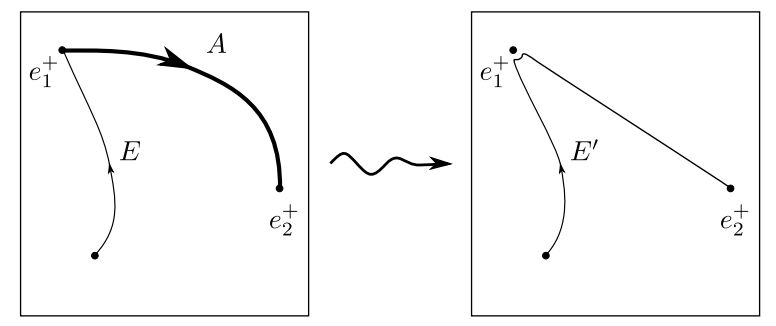

Figure 3.16: A schematic view of the same retrograde saddle connection as in Figure 3.15

diately to the right of $A$ at $o(A)$ with respect to the cyclic ordering of edges of 
$\Gamma_{+} \cup \Gamma^{-}$incident to $o(A)$. So the oriented arc $A$ completely describes the bifurcation. We will denote by $A\left(\Gamma_{+}\right)$the graph obtained from $\Gamma_{+}$after a bifurcation described by $A$ (up to isotopy).

Returning to the codimension 2 bifurcation at $\left(s_{0}, t_{0}\right)$ we have two distinct strata $\Sigma_{\mathrm{sc}}^{1}\left(A_{1}\right)$ and $\Sigma_{\mathrm{sc}}^{1}\left(A_{2}\right)$ corresponding to distinct (oriented) bifurcation arcs $A_{1}$ and $A_{2}$, see Figure 3.17. We take the graph $\Gamma_{+}$of the Bottom region as a

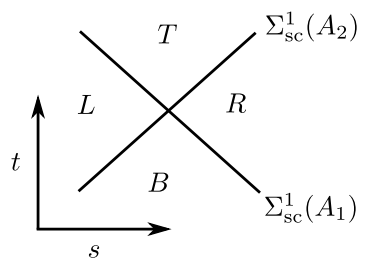

Figure 3.17: Regions in the parameter space.

reference and apply to it the following proposition. Note that, on a tree, any ordered pair of vertices determines a unique oriented segment.

Proposition 38. Suppose $\Gamma$ is a tree and $A_{1}$ and $A_{2}$ are bifurcation arcs for $\Gamma$. The following properties are equivalent.

1. $A_{1}(\Gamma)$ is not a tree but $A_{2}\left(A_{1}(\Gamma)\right)$ is a tree.

2. On $\Gamma$, the oriented segment $S$ from $d\left(A_{2}\right)$ to $d\left(A_{1}\right)$ contains, in that order: $d\left(A_{2}\right) \leq o\left(A_{1}\right)<o\left(A_{2}\right) \leq d\left(A_{1}\right)$ and, furthermore, $S$ is immediately to the right of $A_{1}$ at $o\left(A_{1}\right)$ and $A_{2}$ at $o\left(A_{2}\right)$.

Note that condition 1 above holds if $\Gamma$ is the tree $\Gamma_{+}$coming from the Bottom region $B$ since we assume $T$ and $B$ are in $\Omega_{c}$ while $R$ is in $\Omega_{d}$. This proposition concludes the proof of Theorem 37 because condition 2 above is symmetric in $A_{1}$ and $A_{2}$ (here one should not forget that exchanging $A_{1}$ and $A_{2}$ will reverse the orientation on $S)$. So the graph $A_{2}(\Gamma)$ corresponding to the left region $L$ is not a tree and $L$ is also in $\Omega_{d}$.

Proof. We first prove that property 1 implies property 2. Let $E$ be the edge of $\Gamma$ modified by $A_{1}$. In particular $E$ has vertices $o\left(A_{1}\right)$ and some other vertex $v$ and $E$ is immediately to the right of $A_{1}$ at $o\left(A_{1}\right)$. Because $\Gamma$ is a tree, $v$ can't be the same as $o\left(A_{1}\right)$ and (the closure of) $\Gamma \backslash E$ is the disjoint union of two trees $\Gamma_{1}$ containing $o\left(A_{1}\right)$ and $\Gamma_{2}$ containing $v$, see Figure 3.18

Note that $d\left(A_{1}\right)$ cannot be in $\Gamma_{1}$ since otherwise $A_{1}(E)$ would go from $\Gamma_{1}$ to $\Gamma_{2}$ and $A_{1}(\Gamma)$ would be a tree.

So $d\left(A_{1}\right)$ is in $\Gamma_{2}$ and this implies that $v$ in the segment $\left[o\left(A_{1}\right), d\left(A_{1}\right)\right] \subset \Gamma$. Also we learn that $A_{1}(\Gamma)$ is the disjoint union of the tree $\Gamma_{1}$ and the graph $\Gamma_{2} \cup A_{1}(E)$ which contains exactly one cycle $C$. This cycle contains $A_{1}(E)$ and its vertices are all in $\left[v, d\left(A_{1}\right)\right] \subset \Gamma$, see Figure 3.18 again.

Since $A_{2}\left(A_{1}(\Gamma)\right)$ is a tree, the edge $E^{\prime}$ modified by $A_{2}$ in $A_{1}(\Gamma)$ belongs to $C$ otherwise $C$ would persist in $A_{2}\left(A_{1}(\Gamma)\right)$. So we get that $o\left(A_{2}\right)$ is in $C$ (in particular it can't be the same as $\left.o\left(A_{1}\right)\right)$. In addition $d\left(A_{2}\right)$ is in $\Gamma_{1}$ otherwise $A_{2}\left(A_{1}(\Gamma)\right)$ would stay disconnected. The last thing to check is that $E^{\prime}$ is part of the segment $\left[d\left(A_{2}\right), d\left(A_{1}\right)\right] \subset \Gamma$. The only edge of $C$ which is not in this 

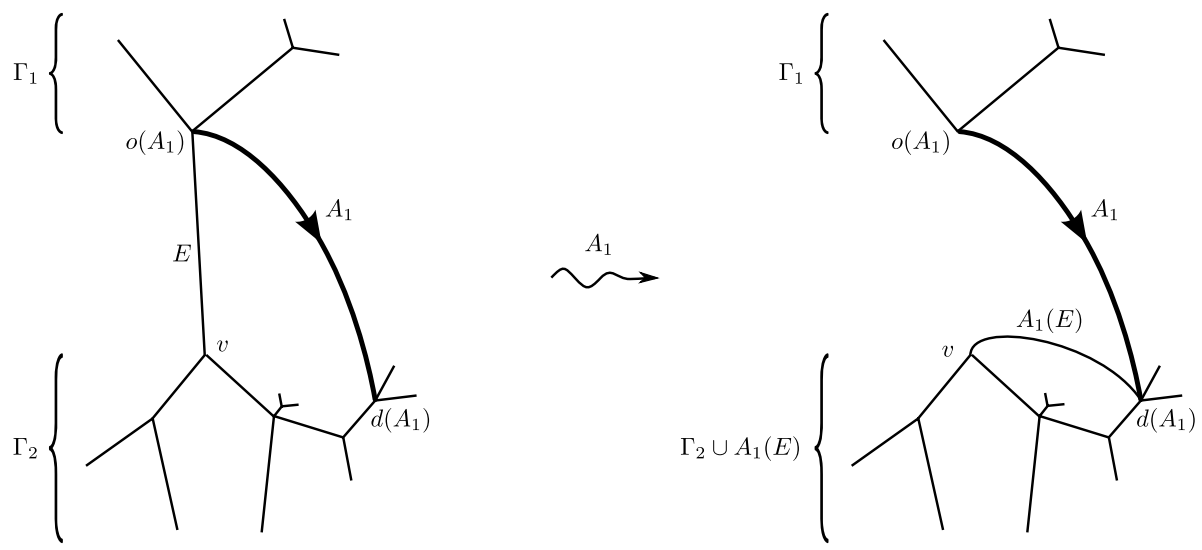

Figure 3.18: Trees and graphs in the proof of Propostion 38

segment is $A_{1}(E)$. Remember $E^{\prime}$ is immediately to the right of $A_{2}$ at $o\left(A_{2}\right)$ so it cannot be $A_{1}(E)$ because that would force $A_{2}$ to go into the disk bounded by $C$ which does not contain $\Gamma_{1}$ (surreptitiously using Schönflies theorem again).

We now prove the converse implication. Since $S$ is immediately to the right of $A_{1}$ at $o\left(A_{1}\right)$, it contains the edge $E$ of $\Gamma$ moved by $A_{1}$. More precisely, $E$ is in the segment $\left[o\left(A_{1}\right), d\left(A_{1}\right)\right] \subset \Gamma$. So $A_{1}(\Gamma)$ is the disjoint union of a tree $\Gamma_{1}$ and a graph $\Gamma_{2}$ containing a unique cycle $C$. Since $S$ is immediately to the right of $A_{2}$ at $o\left(A_{2}\right)$ and $o\left(A_{1}\right) \neq o\left(A_{2}\right)$, the edge $E^{\prime}$ in $A_{1}(\Gamma)$ moved by $A_{2}$ is either an edge in $S$ or $A_{1}(E)$. In both cases, it is contained in $C$. So the cycle $C$ does not persist in $A_{2}\left(A_{1}(\Gamma)\right)$ and $A_{2}\left(E_{1}\right)$ connects $\Gamma_{2} \backslash E^{\prime}$ to $\Gamma_{1}$. Hence $A_{2}\left(A_{1}(\Gamma)\right)$ is a tree.

Now this proof is finished let us see where we used the contact condition and not only properties of generic families of foliations with two parameters. The first thing is that $\Sigma^{1}$ is transverse to the $t$ direction because of the bifurcation lemmas. A second more subtle point is that the crossing lemma says more: it tells the direction of the bifurcations: separatrices turn to their right when $t$ increases. Figure 3.19 show how the above proof would fail if $A_{1}$ and $A_{2}$ were allowed to act as switches in opposite direction. In that figure one sees an example of the bad situation of Figure 3.14. The explanation is that, if we assume that the bifurcation corresponding to $A_{1}$ acts in the wrong direction then, in Proposition 38, we must replace "to the right of $A_{1}$ " by "to the left of $A_{1}$ " and we loose symmetry between $A_{1}$ of $A_{2}$. Of course if both $A_{1}$ and $A_{2}$ act in the wrong direction then we do not have any difference, this simply corresponds to considering negative tight contact structures on $\mathbb{S}^{3}$.

\section{References}

[Ben83] D. Bennequin, Entrelacements et équations de Pfaff, Third Schnepfenried geometry conference, Vol. 1 (Schnepfenried, 1982), 1983, pp. 87161. 


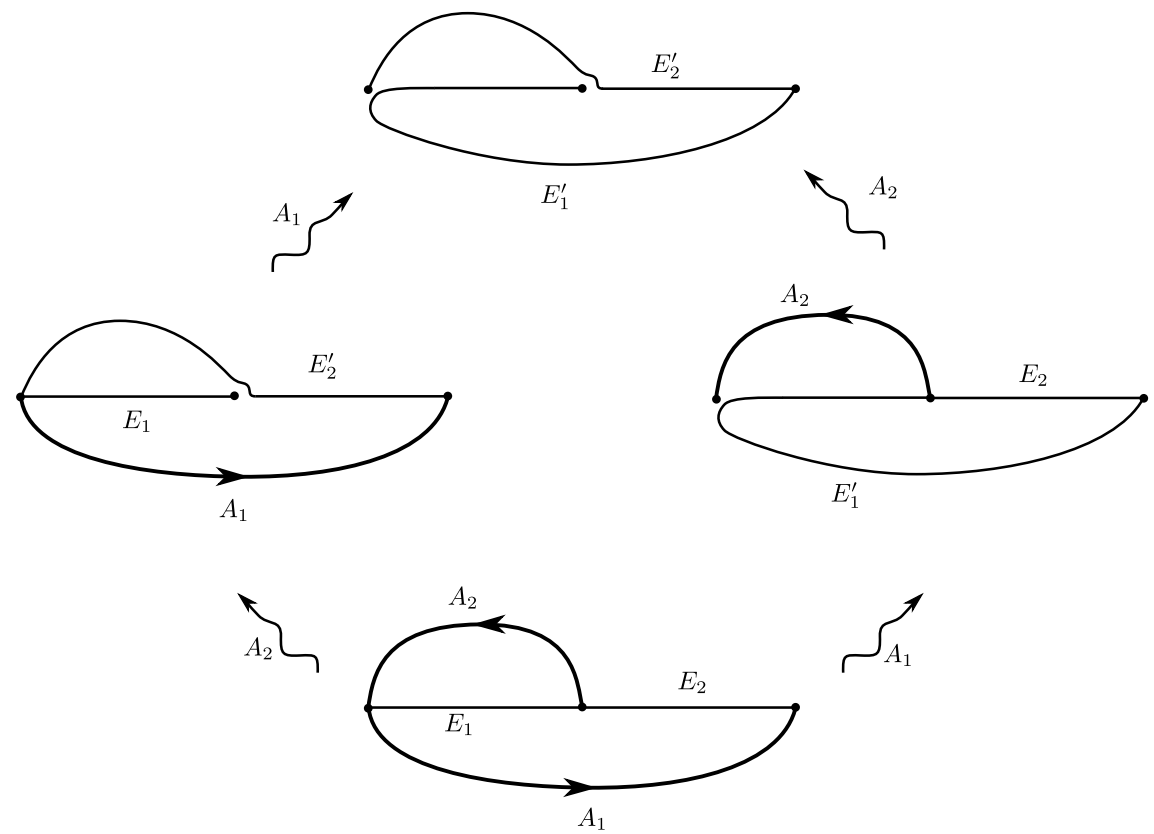

Figure 3.19: How the discussion would fail if $A_{1}$ were reversed. In this example the reference graph has three vertices and two edges. Regions $L, T$ and $B$ are tight whereas $R$ is overtwisted.

[CGH09] V. Colin, E. Giroux, and K. Honda, Finitude homotopique et isotopique des structures de contact tendues, Publ. Math. Inst. Hautes Études Sci. 109 (2009), 245-293, DOI 10.1007/s10240-009-0022-y (French, with French summary).

[Eli89] Y. Eliashberg, Classification of overtwisted contact structures on 3manifolds, Invent. Math. 98 (1989), no. 3, 623-637.

[Eli92] _ Contact 3-manifolds twenty years since J. Martinet's work, Ann. Inst. Fourier (Grenoble) 42 (1992), no. 1-2, 165-192.

[Eli91] _ New invariants of open symplectic and contact manifolds, J. Amer. Math. Soc. 4 (1991), no. 3, 513-520, DOI 10.2307/2939267. MR1102580 (92c:58030)

[Etn04] J. Etnyre, Convex surfaces in contact geometry (2004), available at http://people.math.gatech.edu/ ${ }^{\text {etnyre/preprints/papers/ }}$ surfaces.pdf

[Gei08] H. Geiges, An introduction to contact topology, Cambridge Studies in Advanced Mathematics, vol. 109, Cambridge University Press, Cambridge, 2008.

[Gir91] E. Giroux, Convexité en topologie de contact, Comment. Math. Helv. 66 (1991), no. 4, 637-677.

[Gir00] _ Structures de contact en dimension trois et bifurcations des feuilletages de surfaces, Invent. Math. 141 (2000), no. 3, 615-689. 
[Gir01a] _ Structures de contact sur les variétés fibrées en cercles audessus d'une surface, Comment. Math. Helv. 76 (2001), no. 2, 218262 .

[Gir01b] _ Sur les transformations de contact au-dessus des surfaces, Essays on geometry and related topics, Vol. 1, 2, Monogr. Enseign. Math., vol. 38, Enseignement Math., Geneva, 2001, pp. 329-350 (French).

[Gra59] J. W. Gray, Some global properties of contact structures, Ann. of Math. (2) 69 (1959), 421-450.

[Hon] K. Honda, Contact geometry, available at http://www-bcf.usc.edu/ $\sim$ khonda/math599/notes.pdf.

[Lib59] P. Libermann, Sur les automorphismes infinitésimaux des structures symplectiques et des structures de contact, Colloque Géom. Diff. Globale (Bruxelles, 1958), Centre Belge Rech. Math., Louvain, 1959, pp. 37-59 (French).

[Mor11] A. Mori, On the violation of Thurston-Bennequin inequality for a certain non-convex hypersurface (2011), available at http://arxiv.org/ abs $/ 1111.0383$

[PdM82] J. Palis Jr. and W. de Melo, Geometric theory of dynamical systems, Springer-Verlag, New York, 1982. An introduction; Translated from the Portuguese by A. K. Manning.

[Sot74] J. Sotomayor, Generic one-parameter families of vector fields on two-dimensional manifolds, Inst. Hautes Études Sci. Publ. Math. 43 (1974), 5-46.

Université Paris Sud, 91405 Orsay, France

Email adress: patrick.massot@math.u-psud.fr

$U R L$ : www.math.u-psud.fr/ pmassot/ 